\title{
Regulation of yeast fatty acid desaturase in response to iron deficiency
}

Antonia María Romero*,1, Tania Jordá,,1, Nicolas Rozès², María Teresa Martínez-Pastor ${ }^{3}$ and Sergi Puig ${ }^{1, \#}$

${ }^{1}$ Departamento de Biotecnología, Instituto de Agroquímica y Tecnología de Alimentos (IATA), Consejo Superior de Investigaciones Científicas (CSIC), Paterna, Valencia, Spain.

${ }^{2}$ Departament de Bioquímica i Biotecnología, Facultat d'Enologia, Universitat Rovira i Virgili, Tarragona, Spain.

${ }^{3}$ Departamento de Bioquímica y Biología Molecular, Universitat de València, Burjassot, Valencia, Spain.

*These authors contributed equally to this work and should be considered cofirst authors.

\# To whom correspondence should be addressed:

Sergi Puig, Dept. Biotecnología, IATA-CSIC, Agustín Escardino 7, 46980, Paterna, Valencia, Spain. Tel: (+34) 963900 022. Fax: (+34) 963636301. Email: spuig@iata.csic.es. 


\section{Abstract}

Unsaturated fatty acids (UFA) are essential components of phospholipids that greatly contribute to the biophysical properties of cellular membranes. Biosynthesis of UFAs relies on a conserved family of iron-dependent fatty acid desaturases, whose representative in the model yeast Saccharomyces cerevisiae is Ole1. OLE1 expression is tightly regulated to adapt UFA biosynthesis and lipid bilayer properties to changes in temperature, and in UFA or oxygen availability. Despite iron deficiency being the most extended nutritional disorder worldwide, very little is known about the mechanisms and the biological relevance of fatty acid desaturases regulation in response to iron starvation. In this report, we show that endoplasmic reticulum-anchored transcription factor Mga2 activates OLE1 transcription in response to nutritional and genetic iron deficiencies. Cells lacking MGA2 display low UFA levels and do not grow under iron-limited conditions, unless UFAs are supplemented or OLE1 is overexpressed. The proteasome, E3 ubiquitin ligase Rsp5 and the Cdc48 ${ }^{\text {Npl4/Ufd1 }}$ complex are required for OLE1 activation during iron depletion. Interestingly, Mga2 also activates the transcription of its own mRNA in response to iron deficiency, hypoxia, low temperature and low UFAs. MGA2 up-regulation contributes to increase OLE1 expression in these situations. These results reveal the mechanism of OLE1 regulation when iron is scarce and identify the MGA2 auto-regulation as a potential activation strategy in multiple stresses. 


\section{Highlights (3-5 sentences with max. 85 characters including spaces)}

1) Mga2 activates OLE1 $\triangle 9$-FA desaturase transcription in response to iron deficiency

2) OLE1 activation by Mga2 is vital under low iron conditions unless UFAs are added

3) Defects in UFA biosynthesis end in OLE1 activation in response to low iron conditions

4) OLE1 up-regulation by low iron requires the proteasome, Rsp5, Npl4 and Ufd1 proteins

5) Mga2 activates its own transcription in response to low iron, temperature and oxygen

Keywords: yeast, Saccharomyces cerevisiae, iron deficiency, fatty acids, Ole1, Mga2, hypoxia, cold.

Abbreviations: BPS, bathophenanthroline disulfonic acid disodium; ChIP, chromatin immunoprecipitation; ER, endoplasmic reticulum; FA, fatty acid; FAS, ferrous ammonium sulfate; Pol II, polymerase II; OD, optical density; RT-qPCR, Reverse Transcription-quantitative real time Polymerase Chain Reaction; SFA; saturated fatty acid; TM, transmembrane; TR, transcription rate; UFA; unsaturated fatty acid. 


\section{Introduction}

Cellular membranes are an essential requirement for life. In eukaryotic organisms, membranes represent selective and dynamic boundaries from the environment and between different intracellular compartments. Biological membranes are composed mostly of proteins and lipids, and glycerophospholipids have a major impact on their biophysical properties. The composition of membranes varies among organisms, cellular types and intracellular organelles to achieve defined properties and functions. Cells adjust the proportion of the saturated fatty acids (SFAs) and unsaturated fatty acids (UFAs) of their lipid bilayers in response to multiple environmental stresses to maintain proper fluidity, lipid packing and water permeability. Defects in UFA production profoundly reorganize organelle abundance and morphology, which can lead in extreme cases to cell death. Furthermore, UFA overproduction may be harmful, limit cell division and cause necrosis (reviewed in (Ballweg and Ernst, 2017)). Therefore, UFA biosynthesis has to be tightly regulated. In humans, lipid metabolism deregulation has been linked to obesity-induced morbidity, type II diabetes, schizophrenia, Alzheimer's disease and Parkinson's disease (Aguilar and de Mendoza, 2006; Adiphatla and Hatcher, 2007).

The budding yeast Saccharomyces cerevisiae has been used as a reliable model organism to study multiple aspects of eukaryotic lipid biology (Singh, 2017). The yeast Ole1 $\Delta 9$-fatty acid desaturase (the denoted SCD family in mammals) is a conserved iron- and oxygen-dependent enzyme anchored to the endoplasmic reticulum (ER) membrane that catalyzes the irreversible de novo biosynthesis of mono-UFAs [palmitoleic (16:1) and oleic (18:1)], which represent more than $70 \%$ of total fatty acids (FAs), from the 
corresponding CoA-activated SFAs [palmitic (16:0) and stearic (18:0)]. Ole1 function is essential in yeast, unless the medium is supplemented with UFAs (reviewed in (Aguilar and de Mendoza, 2006; Covino et al., 2016; Martin et al., 2007)). OLE1 expression is highly regulated by numerous stimuli, including carbon source, extracellular FAs, temperature, oxygen levels and metal ions. Thus OLE1 expression increases in response to low oxygen and low temperature, but is suppressed by UFA supplementation (Chellappa et al., 2001; Hoppe et al., 2000; Kwast et al., 1999; Nakagawa et al., 2002; Vasconcelles et al., 2001). Two homologous and partially redundant transcription factors, Spt23 and Mga2, regulate OLE1 expression. They are synthesized as inactive $\sim 120 \mathrm{KDa}$ homodimeric precursors anchored to the ER membrane through their carboxy-terminal transmembrane (TM) helix domain. Proteolytic cleavage releases a $\sim 90 \mathrm{KDa}$ amino-terminal fragment that translocates to the nucleus and activates OLE1 transcription in a response denoted as the OLE pathway (Hoppe et al., 2000; Piwko and Jentsch, 2006). Although some differences exist, the activation of both transcription factors requires specific ubiquitylation by E3 ligase Rsp5, processing by the proteasome, and mobilization from the ER membrane with the help of substrate-recruiting factor Ubx2 and segregase/chaperone complex Cdc48 ${ }^{\text {Np14/Ufd1 }}$ (Chellappa et al., 2001; Hitchcock et al., 2001; Hoppe et al., 2000; Kolawa et al., 2013; Rape et al., 2001; Shcherbik and Haines, 2007; Shcherbik et al., 2003; Surma et al., 2013). Whereas the mga2 $\Delta$ and spt23 $\Delta$ single mutants are viable, the mga2 $\Delta s p t 23 \Delta$ double mutant is lethal unless UFAs are added to the growth medium (Chellappa et al., 2001; Zhang et al., 1999). Although each transcription factor is sufficient for OLE1 expression, Mga2 is the 
dominant factor that activates OLE1 in response to hypoxia, low temperature, cobalt and nickel (Chellappa et al., 2001; Jiang et al., 2002; Jiang et al., 2001; Nakagawa et al., 2002). Recent results have demonstrated that dimeric Mga2 functions as the membrane sensor for lipid saturation (Covino et al., 2016). Specifically, Mga2 uses the rotational orientation of its transmembrane helix to sense lipid packing in the ER and to control OLE1 transcription. Thus a high proportion of ER membrane SFAs promotes an Mga2 TM helix rotational orientation that activates the OLE pathway, while an increase in UFA abundance stabilizes inactive Mga2 (Covino et al., 2016).

Budding yeast uses two partially redundant transcription factors, Aft1 and Aft2, to activate the transcription of a group of genes, collectively referred to as the iron regulon, in response to iron deficiency. The iron regulon includes metalloreductases that reduce extracellular $\mathrm{Fe}^{3+}$ to its more soluble $\mathrm{Fe}^{2+}$ form, and the high-affinity iron transport system composed of multicopper ferroxidase Fet3 and iron-permease Ftr1 (reviewed in (Kaplan and Kaplan, 2009; Sanvisens and Puig, 2011)). Yeast cells also express low-affinity iron transporters, such as Fet4 and Smf1. Despite oxo-diiron being an indispensable cofactor for the catalytic activity of $\Delta 9$-fatty acid desaturase Ole1, very little is known about the mechanisms that regulate these enzymes in response to alterations in iron bioavailability and how it affects UFA biosynthesis. We have previously reported that addition of the $\mathrm{Fe}^{2+}$-specific chelator bathophenanthroline disulfonic acid disodium (BPS) to growth medium allows OLE1 transcript levels to increase (Puig et al., 2005). In this study, we present evidence to support that Mga2 activates OLE1 transcription in response to iron limitation. This regulatory mechanism is essential for growth under iron-deficient 
conditions unless UFAs are added to the medium or OLE1 is ectopically expressed. In mechanistic terms, ER-embedded Mga2 transcription factor activation by iron depletion seems a consequence of alterations in the relative

FA composition of yeast membranes. Interestingly, we also reveal an Mga2 transcriptional auto-regulatory mechanism in response to iron deficiency, hypoxia and low temperatures or UFAs that contributes to enhance OLE1 expression.

\section{Material and Methods}

\subsection{Yeast strains, culture conditions, and plasmids}

The yeast strains used in this study are listed in Table 1. We used the pFA6a-13Myc-KanMX6 plasmid as a template (Longtine et al., 1998), and specific oligonucleotides, to generate an integrative cassette for tagging the genomic copy of OLE1 with the 13xMyc epitope at the carboxyl terminus (SPY901 yeast strain). Yeast precultures were incubated overnight at $30^{\circ} \mathrm{C}$ in liquid synthetic complete SC medium $[0.17 \%(\mathrm{w} / \mathrm{v})$ yeast nitrogen base without amino acids and without ammonium sulfate (Pronadisa), $0.5 \%(\mathrm{w} / \mathrm{v})$ ammonium sulfate (Panreac), 2\% (w/v) glucose (Panreac), and $2 \mathrm{~g} / \mathrm{L}$ Kaiser drop-out (Formedium)] lacking specific requirements whenever necessary, and reinoculated at an optical density at $600 \mathrm{~nm}\left(\mathrm{OD}_{600}\right)$ of 0.2 . To regulate iron availability in liquid cultures, cells were incubated for $6 \mathrm{~h}$ at $190 \mathrm{rpm}$ in SC medium (+Fe) or SC supplemented with $100 \mu \mathrm{M}$ BPS (Sigma) $(-\mathrm{Fe})$. The $\mathrm{Fe}^{2+}$ specific chelator ferrozine (Sigma) was used at the indicated concentrations to limit iron bioavailability in $2 \%$ agar (Pronadisa) solid media. Fatty acids (oleic 
and linoleic acids, Sigma) were added to a final $1 \mathrm{mM}$ concentration and stabilized with $1 \%$ Tergitol Nonidet P-40 (Sigma). To induce $P_{\text {GAL1 }}$-OLE1 expression, glucose in SC was replaced with $2 \%$ galactose. For the spot assays, yeast cells were grown to the exponential phase, spotted in 10-fold serial dilutions starting at on $\mathrm{OD}_{600}$ of 0.1 , and incubated at $30^{\circ} \mathrm{C}$ for 3 days. The temperature sensitive strains were cultivated in SC at the permissive temperature of $25^{\circ} \mathrm{C}$ and then transferred to the non permissive temperature of $37^{\circ} \mathrm{C}$. After $1 \mathrm{~h}$ at $37^{\circ} \mathrm{C}, 100 \mu \mathrm{M}$ BPS was added, $(-\mathrm{Fe})$ or not $(+\mathrm{Fe})$, and cells were incubated for $5 \mathrm{~h}$ more. With the $n p / 4-1$ mutant, $30^{\circ} \mathrm{C}$ was used as the restrictive temperature (Hitchcock et al., 2001). For growth at low temperatures, yeast cells were collected, transferred to a pre-cold medium at $10^{\circ} \mathrm{C}$ and incubated for $1 \mathrm{~h}$. Hypoxia was achieved by bubbling nitrogen at a constant pressure for $5 \mathrm{~h}$.

All the plasmids used in this study are listed in Table 1. To construct the pSP1039 plasmid, 470 base pairs were amplified from the promoter region of the MGA2 gene with oligonucleotides MGA2-470F-HindIII and MGA2-lacZ-Pstl$\mathrm{R}$ (see Table 2), and then the PCR product was digested with restriction enzymes Hindlll (Roche) and Pstl (Roche). The YCp33RNR4Z plasmid, previously digested with HindllI and Pstl to remove the RNR4 promoter, was used to clone the MGA2 promoter fused to the lacZ reporter gene. PCR amplifications were performed with Phusion polymerase (Finnzymes), and the cloned insert was sequenced. One Shot TOP10 chemically competent Escherichia coli cells (Invitrogen) were used to isolate and propagate plasmids. 
Table 1. List of yeast strains and plasmids used in this study.

\begin{tabular}{|c|c|c|}
\hline Strain or plasmid & Description & Source \\
\hline \multicolumn{3}{|l|}{ Strains } \\
\hline HTLU-2832-1B & $\begin{array}{l}\text { W303 MATa HIS3, TRP1, LEU2, URA3, } \\
\text { ADE2, can1 }\end{array}$ & F. Cross \\
\hline W303-1A & $\begin{array}{l}\text { MATa, ura3-1, ade2-1, trp1-1, his3-11,15, } \\
\text { leu2-3,112 }\end{array}$ & E. Herrero \\
\hline MML1088 & W303-1A aft1 $\triangle 5$ aft2::KanMX4 & E. Herrero \\
\hline BY4741 & MATa his $3 \Delta 1$ leu2 $\Delta 0$ met $15 \Delta 0$ ura3 $\Delta 0$ & Invitrogen \\
\hline SPY386 & BY4741 fet3::URA3 fet4::KanMX4 & (Sanvisens et al., 20 \\
\hline SPY824 & BY4741 mga2::KanMX4 & Invitrogen \\
\hline SPY823 & BY4741 spt23::KanMX4 & Invitrogen \\
\hline YWO0607 & MATa ura3 leu2-3,112 his3-11,15 Gal & D. H. Wolf \\
\hline YWO0608 & YW00607 pre1-1 & D. H. Wolf \\
\hline YWO1 & $\begin{array}{l}\text { MATa his3- } 4200, \text { leu2-3,112, lys2-801, } \\
\text { trp1-1(am), ura3-52 }\end{array}$ & (Hoppe et al., 2000) \\
\hline Y0356 & YWO1 rsp5::HIS3; ura3-52::RSP5::URA3 & (Hoppe et al., 2000) \\
\hline Y0358 & YWO1 rsp5::HIS3; ura3-52::rps5-2::URA3 & (Hoppe et al., 2000) \\
\hline PSY580 & MATa ura3-52 leu2 $\Delta 1 \operatorname{trp} 1 \Delta 63$ & (Hitchcock et al., 200 \\
\hline PSY2340 & PSY580 np/4-1 & (Hitchcock et al., 200 \\
\hline PSY3074 & $\begin{array}{l}\text { MATa his4-519, ura3-52, ade1-100, leu2- } \\
\text { 3,112 ufd1-1 }\end{array}$ & (Hitchcock et al., 200 \\
\hline SPY901 & BY4741 OLE1-13хMyc::KanMX6 & This study \\
\hline
\end{tabular}




\section{Plasmids}

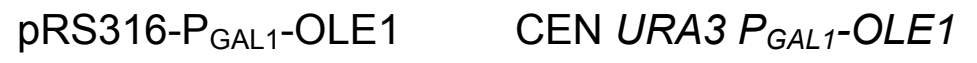

pPS2364

pPS2358

YEplac181-3HA-MGA2

YCp33RNR4Z

pSP1039

pFA6a-13Myc-KanMX6
CEN URA3 MGA2

CEN URA3 MGA2-truncated

$2 \mu$ LEU2 3HA-MGA2

CEN URA3 $P_{R N R 4-l a c Z}$

CEN URA3 $P_{M G A 2-l a c Z}$

Integrative KanMX6
(Hoppe et al., 2000)

(Hitchcock et al., 20C

(Hitchcock et al., 20C

(Hoppe et al., 2000)

R. S. Zitomer

This study

(Longtine et al., $199 \varepsilon$

\subsection{RNA analyses}

Total RNA extraction and cellular mRNA levels were determined by RTqPCR as previously described (Sanvisens et al., 2014). The primers used for RT-qPCR are listed in Table 2. The OLE1 and MGA2 transcription rates (TRs) were determined in triplicate by the Genomic Run-On (GRO) technique as previously reported (Garcia-Martinez et al., 2004). The ArrayStat software (Imaging Research, Inc.) was used to determine reproducibility and the mean of the GRO values.

Table 2. Oligonucleotides used in this work.

\begin{tabular}{ll}
\hline Name & Sequence (from 5' to 3') $^{\prime}$ \\
\hline OLE1-qPCR-F & TCGACAAGAAGGGAAACGAA \\
\hline OLE1-qPCR-R & CATGGTTGTTCGGAGATGTG \\
\hline MGA2-qPCR-F & ATCTGTTCCCGTTGTCTTGG \\
\hline MGA2-qPCR-R & CCTCTTCCTCATAATCCTCTTCCT \\
\hline PGK1-qPCR-F & AAGCGTGTCTTCATCAGAGTTG
\end{tabular}




\begin{tabular}{ll} 
PGK1-qPCR-R & CGTATCTTGGGTGGTGTTCC \\
\hline OLE1-ChIP-prom-287F & CTCAGACACACCTATCCCTATTGT \\
\hline OLE1-ChIP-prom-186R & AGCCAGGAGCCGATGATTT \\
\hline FUS1-ChIP-prom-F & CATGTGGACCCTTTCAAAAC \\
\hline FUS1-ChIP-prom-R & AGACAGCGCGAAAAGTGACA \\
\hline MGA2-470F-HindIII & ACTGAAGCTTCATCAAGAGCGATTGGATGACAGT7 \\
\hline MGA2-lacZ-Pstl-R & AATACTGCAGCATAACGAAATGTTCTGTTCGCCA \\
\hline OLE1-F2 & AAATGA \\
\hline OLE1-R1 & TAGTAAGAGAGGTGAAATCTACGAAACTGGTAAG \\
\hline OLE1+320R & ATCTTTCGGATCCCCGGGTTAATTAA \\
\hline TEF-term:135F & AGTTGAATTCGAGCTCGTTTAAAC \\
\hline
\end{tabular}

\subsection{Protein analyses}

Total protein extracts were obtained by using the alkali method (Kushnirov, 1998). Equal amounts of protein were resolved in SDS-PAGE gels and transferred onto nitrocellulose membranes. The primary antibodies used were anti-c-myc (9E10, Roche) and anti-Pgk1 (22C5D8; Invitrogen). Immunoblots were developed with horseradish peroxidase (HRP)-labeled secondary antibodies and the ECL Select Western blotting detection kit (GE Healthcare Life Sciences). Immunoblot images were obtained with an ImageQuant LAS 4000 mini Biomolecular Imager (GE Healthcare Life Sciences). Data were processed and Ole1-13Myc/Pgk1 protein levels were quantified with ImageQuant TL analysis software (GE Healthcare Life Sciences).

Chromatin immunoprecipitation (ChIP) assays were performed to determine RNA polymerase II binding to the OLE1 and FUS1 promoters as 
previously described (Gomar-Alba et al., 2013). Cell extracts were incubated with Dynabeads Pan Mouse IgG (Invitrogen), previously bound to a monoclonal mouse anti-Rpb1 antibody (clone 8WG16, Covance). DNA was purified with the High Pure PCR Product Purification Kit (Roche). The primers used for the OLE1 and FUS1 promoter RT-qPCR analyses are listed in Table 2.

\subsection{Determination of total fatty acids}

The fatty acid methyl ester analysis was performed following a modified version of a method previously reported, which determines total fatty acids including free and esterified fatty acids of yeast cells (Borrull et al., 2015). First $1 \mathrm{~mL}$ of $\mathrm{HCl} 1.25 \mathrm{~N}$ in methanol, $10 \mu \mathrm{L}$ heptanoic acid $\left(\mathrm{C} 7,1 \mathrm{~g} \mathrm{~L}^{-1}\right)$ and $10 \mu \mathrm{L}$ heptadecanoic acid $\left(\mathrm{C} 17,4 \mathrm{~g} \mathrm{~L}^{-1}\right)$ were added to glass tubes that contained a yeast pellet of around $5 \cdot 10^{8}$ cells. Samples were heated to $90^{\circ} \mathrm{C}$ for 60 min and were then cooled to room temperature. After cooling, $1 \mathrm{~mL}$ of $\mathrm{NaCl} 0.9 \%(\mathrm{w} / \mathrm{v})$ in water and $300 \mu \mathrm{L}$ hexane were added. The extraction was repeated twice. Between each extraction phase, tubes were centrifuged at $3000 \times \mathrm{g}$ for $5 \mathrm{~min}$ to allow the best phase separation. Analytical GC was carried out in an Agilent 5890 connected to an HP Vectra computer with the ChemStation software (Agilent Technologies). The extract $(2 \mu \mathrm{L})$ was injected (splitless, $0.75 \mathrm{~min}$ ) into an FFAP-HP column of $30 \mathrm{~m} \times 250 \mu \mathrm{m} \times 0.25 \mu \mathrm{m}$ phase thickness (Agilent Technologies) with an automatic injector (Agilent). The temperature program shifted from $100^{\circ} \mathrm{C}$ to $240^{\circ} \mathrm{C}(5 \mathrm{~min})$ at $4^{\circ} \mathrm{C} \mathrm{min}^{-1}$. Injector and detector temperatures were $220^{\circ} \mathrm{C}$ and $250^{\circ} \mathrm{C}$, respectively. The carrier gas was helium, applied at $1.2 \mathrm{~mL} \mathrm{~min}{ }^{-1}$. Relative amounts of the given fatty acids were calculated from their respective chromatographic peak areas after normalization with internal standards (C7 or C17). 


\section{5. $\beta$-galactosidase assays}

$\beta$-galactosidase activity was measured as previously described (Puig et al., 2004).

\subsection{Statistical analyses}

To evaluate statistical significance, tailed t-student tests were applied.

The asterisk $\left({ }^{*}\right)$ indicates statistically significant differences with $p$-value $<0.05$. 


\section{Results}

\subsection{OLE1 is transcriptionally activated in response to iron deficiency.}

The expression of OLE1, which encodes the yeast $\Delta 9$-fatty acid desaturase, increases upon the addition of iron chelators, such as bathophenanthroline disulfonic acid disodium (BPS) and 1,10-phenanthroline ((Puig et al., 2005; Vasconcelles et al., 2001); Figure 1A). To ascertain whether OLE1 up-regulation was due to iron deficiency and not to a secondary effect of the iron chelators, we performed the following assays. Firstly, we added an excess of ferrous ammonium sulfate (FAS) to the growth medium that contained BPS in order to restore iron bioavailability. In this case, OLE1 mRNA levels dropped to the levels observed for the iron-sufficient conditions (Figure 1A). Secondly, OLE1 transcript levels were determined in the yeast fet3 $\triangle$ fet $4 \Delta$ mutant, which lacked the Fet3 high-affinity and Fet4 low-affinity iron transporters. As shown in Figure $1 \mathrm{~A}$, the fet $3 \Delta$ fet $4 \Delta$ cells grown under ironsufficient conditions displayed higher OLE1 mRNA levels compared to the wildtype cells grown under the same conditions. Thirdly, we assessed the state of hypoxia in yeast cells treated with BPS by determining the levels of two mRNAs, TIR3 and HEM13, which are up-regulated in response to low oxygen (Ter Linde and Steensma, 2002; Kwast et al., 2002). TIR3 encodes a cell wall mannoprotein required for growth under anaerobic conditions, and HEM13 encodes the coproporphyrinogen III oxidase within the heme biosynthetic pathway. The mRNA levels of both genes increased in response to low oxygen, but not upon iron depletion (Figure 1B). Therefore, both hypoxia markers indicated that the addition of BPS did not lead to hypoxia. Finally, to ascertain 
whether the increase in OLE1 mRNAs turned into more Ole1 protein, we epitope tagged Ole1 with $13 x$ Myc at its carboxyl terminus, and we determined its protein levels by Western blot in iron sufficient $(+\mathrm{Fe})$ and iron deficient $(-\mathrm{Fe})$ conditions. As shown in Figure 1C, Ole1 protein levels increased approximately 3.6-fold upon iron depletion. These results strongly suggest that OLE1 expression specifically increases in response to both nutritional and genetic iron deficiencies.

To assess whether the up-regulation of the OLE1 mRNA levels by iron scarcity takes place at the transcriptional level, the Genomic Run-On approach was followed, which determines the transcription rate (TR) of specific genes (Garcia-Martinez et al., 2004). For this purpose, the wild-type cells were cultivated in the presence of $100 \mu \mathrm{M}$ of the $\mathrm{Fe}^{2+}$ chelator BPS for $6 \mathrm{~h}$. Both the OLE1 steady-state mRNA levels and TR increased while iron deficiency progressed, especially after 3 and $6 \mathrm{~h}$ of depletion (Figure 1D). These results demonstrate that OLE1 becomes transcriptionally activated when iron bioavailability is restricted, which increases mRNA levels. 
A

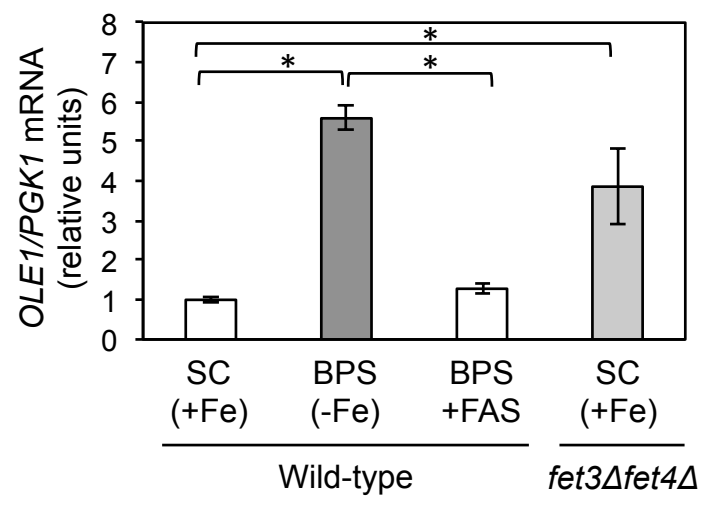

C

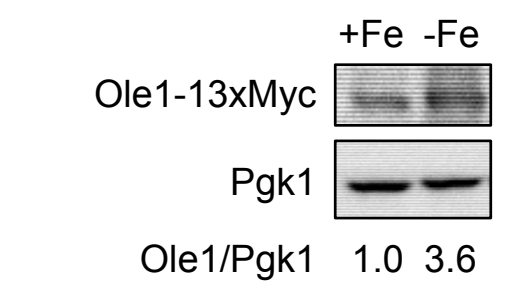

$E$
B

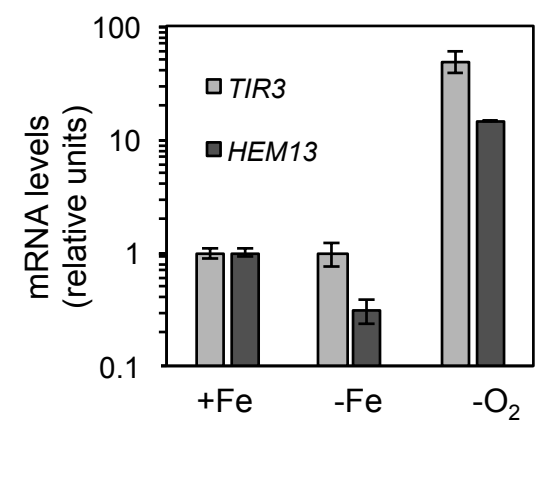

D

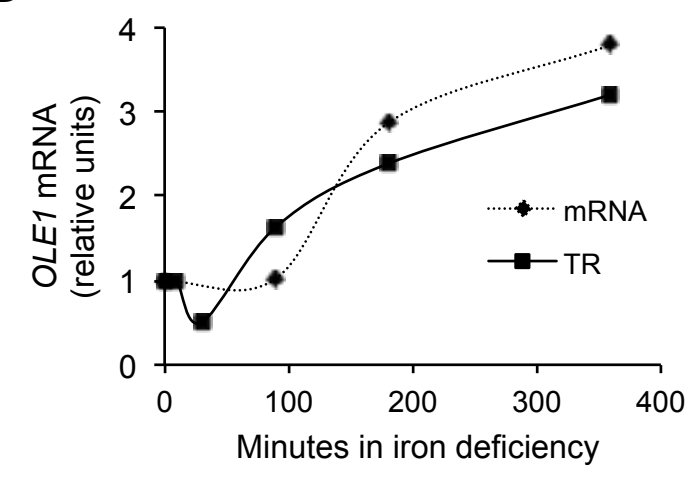

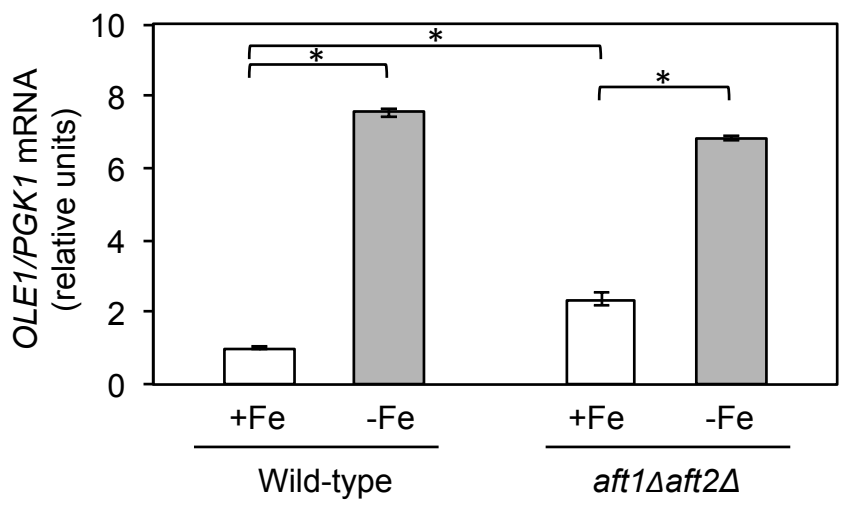

Figure 1. Regulation of the yeast $O L E 1$ mRNA and protein levels by iron bioavailability. (A) OLE1 mRNA levels increased in response to nutritional and genetic iron deficiency. The wild-type (BY4741) and fet34fet44 (SPY386) yeast cells were grown at $30^{\circ} \mathrm{C}$ for $6 \mathrm{~h}$ to the exponential phase in $\mathrm{SC}(+\mathrm{Fe}), \mathrm{SC}$ supplemented with $100 \mu \mathrm{M}$ BPS (-Fe), and SC with $100 \mu \mathrm{M}$ BPS and $300 \mu \mathrm{M}$ ferrous ammonium sulfate or FAS $(-\mathrm{Fe})$. Total RNA was extracted, and the OLE1 mRNA levels were determined by RT-qPCR as described in Material and Methods. (B) The iron chelator BPS does not 
activate hypoxia markers. The wild-type (BY4741) cells were grown at $30^{\circ} \mathrm{C}$ to the exponential phase in SC (+Fe), SC with $100 \mu \mathrm{M}$ BPS $(-\mathrm{Fe})$, and in hypoxic conditions for $5 \mathrm{~h}\left(-\mathrm{O}_{2}\right)$. Total RNA was extracted, and the TIR3 and HEMI3 mRNA levels were determined by RT-qPCR. Transcript levels were normalized with PGK1 mRNA. (C) Ole1 protein levels increase in response to iron depletion. Yeast dells expressing OLE1$13 x M y c$ (SPY901) were grown to exponential phase in SC $(+\mathrm{Fe})$ and $\mathrm{SC}$ with $100 \mu \mathrm{M}$ BPS $(-\mathrm{Fe})$. Total proteins were extracted, and Ole1-13myc and Pgk1 protein levels were determined by immunoblotting with anti-c-myc and anti-Pgk1 antibodies, respectively. Pgk1 was used as a loading control. A representative experiment of two biological samples is shown. Ole1/Pgk1 protein quantification is shown relative to the iron-sufficient conditions. (D) Iron deficiency increased the $O L E 1$ transcription rate. The wild-type (HTLU-2832-1B) cells were grown at $30^{\circ} \mathrm{C}$ in $\mathrm{SC}$ to the early exponential phase (time zero). Then aliquots were isolated $0,10,30,90,180$ and 360 min after adding $100 \mu \mathrm{M}$ BPS. Samples were processed to determine the OLE1 mRNA levels (mRNA) by RT-qPCR and the OLE1 transcription rate (TR) by GRO. (E) Aft1 and Aft 2 were not responsible for $O L E 1$ activation by iron depletion. The wild-type (W303-1A) and aft1 $\Delta$ aft2 (MML1088) cells were grown and analyzed as in panel A. Data represent the average (and standard deviation) of three biologically independent experiments. The asterisk $(*)$ indicates statistically significant differences (p-value $<$ 0.05). For more details, check the Material and Methods section.

The iron-regulated Aft1 and Aft2 transcription factors activate the expression of multiple genes in response to iron deficiency. To determine whether Aft1 and Aft2 participate in OLE1 transcriptional activation by iron depletion, the OLE1 mRNA levels were determined in a wild-type and an 
aft1 $\Delta$ aft2 $2 \Delta$ mutant strain cultivated under iron-sufficient (+Fe) and iron-deficient $(-\mathrm{Fe})$ conditions. We observed that in both strains, the OLE1 mRNA levels increased when iron was limited (Figure 1E), which suggests that Aft1 and Aft2 are not the transcription factors directly responsible for OLE1 regulation by iron. Interestingly, the OLE1 mRNA levels augmented in the aft1 $\triangle$ aft2 $\Delta$ cells compared to a wild-type strain grown under iron-sufficient conditions (Figure 1E). This observation is consistent with the genetic iron deficiency displayed by the aft1 $\Delta$ aft2 $\Delta$ yeast cells, and reinforces that the Aft1- and Aft2-independent OLE1 up-regulation is induced by iron scarcity.

\subsection{Mga2 activates OLE1 expression in response to iron deficiency.}

Previous results have demonstrated that the addition of fatty acids lowers OLE1 mRNA levels (Nakagawa et al., 2002; Vasconcelles et al., 2001). Consistently with this, addition of linoleic acid dramatically diminished OLE1 expression under iron-sufficient conditions (Figure 2A). More importantly, iron deficiency did not activate OLE1 expression when the medium was supplemented with linoleic acid (Figure 2A). This result suggests that changes in total fatty acid composition could influence OLE1 regulation by iron (see below).

A drop in temperature or in the availability of oxygen or UFAs alters the fatty acid composition of membranes and promotes the release of transcription factors Mga2 and Spt23 from the ER membrane, which eventually activate OLE1 transcription (Chellappa et al., 2001; Hoppe et al., 2000; Jiang et al., 2002; Jiang et al., 2001; Nakagawa et al., 2002). To ascertain whether they 
also participate in OLE1 regulation by iron, OLE1 mRNA levels were determined in the wild-type, mga2 $\Delta$, and spt $23 \Delta$ cells cultivated under $+\mathrm{Fe}$ and -Fe conditions. We observed that most of OLE1 mRNA increase was abolished in the cells that lacked MGA2 (Figure 2A). However, only a slight decrease in OLE1 up-regulation was noted in the spt23 $\Delta$ mutant (Figure 2A). We were unable to investigate the OLE1 mRNA levels in those cells that lacked both regulators because the mga2 $\Delta s p t 23 \Delta$ double mutant is not viable (Chellappa et al., 2001; Zhang et al., 1999). Although an Spt23 secondary role cannot be ruled out, these results suggest that Mga2 is the main factor to promote OLE1 activation when iron is scarce.

A

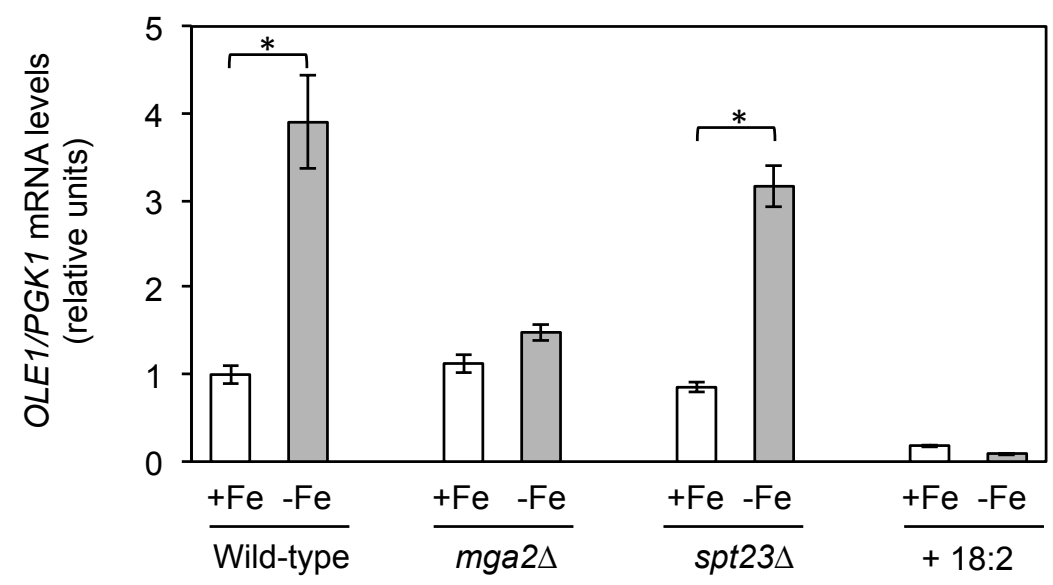

$\mathrm{B}$

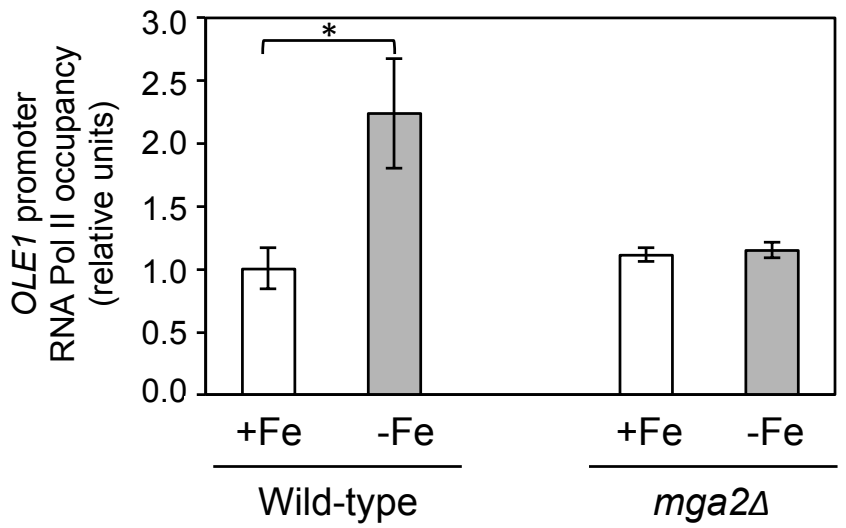


Figure 2. Mga2 activates $O L E 1$ expression under low iron conditions. (A) Regulation of the OLE1 mRNA levels by iron in the mga2A and spt23A mutants. The wild-type (BY4741) cells were grown at $30^{\circ} \mathrm{C}$ for $6 \mathrm{~h}$ in $\mathrm{SC}(+\mathrm{Fe})$ or $\mathrm{SC}+100 \mu \mathrm{M}$ BPS $(-\mathrm{Fe})$ without or with $1 \mathrm{mM}$ linoleic acid (+18:2). The yeast mga24 (SPY824) and spt234 (SPY823) mutants were grown and analyzed as described in Figure 1A. (B) Mga2 facilitated the recruitment of RNA Pol II to the $O L E 1$ promoter in response to iron limitation. The wild-type (BY4741) and mga2d (SPY824) cells were grown as described in Figure 1A. Proteins were extracted and immunoprecipitated with anti-RNA Pol II monoclonal antibody, and binding to $O L E 1$ promoter region was determined by RT-qPCR. The results are shown in relation to the wild-type $+\mathrm{Fe}$ samples and were normalized to FUS1 promoter. Data represent the average and standard deviation of three biologically independent experiments. The asterisk (*) indicates statistically significant differences ( $\mathrm{p}$-value $<0.05)$.

To further test the transcriptional regulation of OLE1 by iron and the implication of Mga2 in this process, we studied the recruitment of RNA polymerase II (Pol II) to the OLE1 locus by chromatin immunoprecipitation (ChIP) in both the wild-type and mga2 $\Delta$ cells grown under $+\mathrm{Fe}$ and $-\mathrm{Fe}$ conditions. Consistently with the increase noted in OLE1 TR obtained when iron was depleted (Figure 1B), we observed that iron deficiency stimulated the occupancy of the OLE1 promoter by RNA Pol II (Figure 2B). It is noteworthy that RNA Pol II recruitment to the OLE1 promoter promoted by iron depletion was not observed in an mga2 $\Delta$ mutant (Figure 2B). These results support the notion that Mga2 activates OLE1 transcription in response to iron deficiency by enhancing the recruitment of RNA Pol II. 


\subsection{Mga2 is essential for growth under low iron conditions due to defects in OLE1 expression and unsaturated fatty acid synthesis}

A large-scale research work has recently shown that yeast mga2 $\Delta$ cells display a growth defect in the presence of the $\mathrm{Fe}^{2+}$ chelator Ferrozine (Samanfar et al., 2013). By adding BPS, we corroborated that mga2 $\Delta$ exhibited complete lack of growth under iron-deficient conditions (Figure 3A). Consistently with our previous results, the spt23 $\Delta$ mutant did not display any growth defect for the iron-deficient conditions compared to the wild-type strain (Figure 3A). To investigate the reasons that underlie the mga2 $\Delta$ phenotype in low iron media, we attempted to rescue it by expressing OLE1 under the control of the galactose inducible and glucose repressed GAL1 promoter ( $P_{G A L 1}$-OLE1 construct). Addition of galactose to the mga2 $\Delta$ cells that expressed $P_{G A L 1}-O L E 1$ rescued their growth defect under iron-deficient conditions (Figure 3B). To further study mga2A sensitivity to iron depletion, we ascertained whether supplementation with UFAs could also rescue growth. Indeed addition of either oleic acid (18:1) or linoleic acid (18:2), which cannot be synthesized by $S$. cerevisiae, but incorporates into the membrane lipids, recovered the growth defect displayed by the mga2 $\Delta$ cells in low iron (Figure 3A). Collectively, these results indicate that mga2 $\Delta$ cells possess defects in UFA synthesis under irondeficient conditions, probably due to lack of OLE1 induction that causes a severe growth defect. 
A

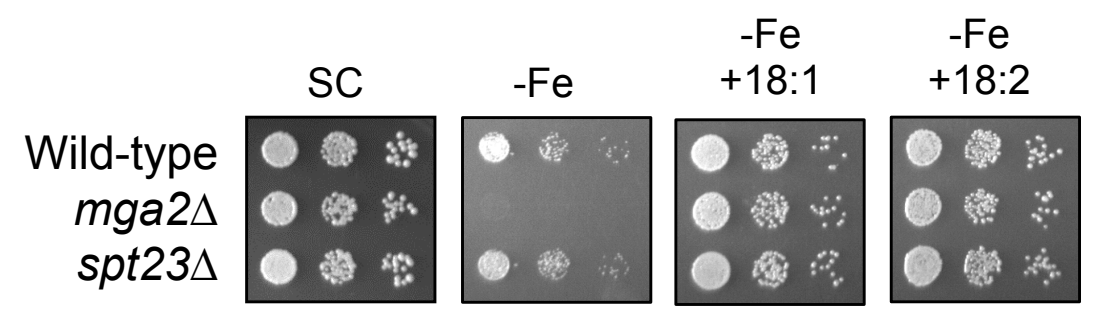

B

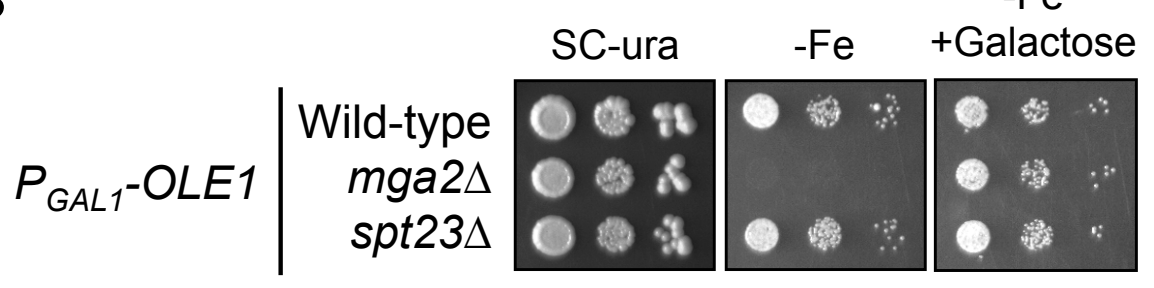

Figure 3. Requirements for growth under iron-deficient conditions. (A) Addition of UFAs rescued mga2 4 growth defect in low iron. The wild-type (BY4741), mga2A (SPY824) and spt234 (SPY823) yeast strains were grown to the exponential phase and spotted in 10-fold serial dilutions on SC and SC with $400 \mu \mathrm{M}$ Ferrozine (-Fe). Oleic $(+18: 1)$ or linoleic $(+18: 2)$ acids were added to - Fe plates to a final $1 \mathrm{mM}$ concentration. (B) The wild-type (BY4741), mga24 (SPY824) and spt234 (SPY823) cells transformed with the pRS316-P $\mathrm{FAL}_{\mathrm{GA}}-\mathrm{OLE} 1$ plasmid were grown to the exponential phase and spotted in 10-fold serial dilutions on SC-ura, SC-ura with $500 \mu \mathrm{M}$ Ferrozine (-Fe), and SC-ura without glucose, but with $2 \%$ galactose and $500 \mu \mathrm{M}$ Ferrozine $(-\mathrm{Fe}+$ Galactose). Plates were incubated for 3 days at $30^{\circ} \mathrm{C}$ and photographed. A representative experiment of at least two independent biological replicates is shown.

As the complementation assays shown herein suggest that the mga2 $\Delta$ mutant could suffer from defects in UFA synthesis under iron-deficient conditions, we decided to determine the total fatty acid (FA) composition of the wild-type and mga2 $\Delta$ cells grown under $+\mathrm{Fe}$ and $-\mathrm{Fe}$ conditions. We observed 
that the percentage of total UFA in relation to SFA lowered in a wild-type strain when cultivated in an iron-limited medium (Figure 4). A decrease in total UFA levels was also observed for a genetic deficiency achieved by using the fet3 $\Delta$ fet4 $\Delta$ strain (Figure 4). These results suggest that UFA synthesis decreases when iron bioavailability is limited either nutritionally or genetically, probably due to a drop in Ole1 FA desaturase activity, which depends on iron. The mga2 $\Delta$ mutant displayed a similar UFA percentage to the wild-type strain under iron-sufficient conditions (Figure 4A), which is consistent with an unaltered OLE1 expression (Figure 2A). However, the decreased UFA abundance displayed by the mga2 $\Delta$ mutant upon iron depletion was much more severe than that observed in a wild-type strain (Figure 4A). Probably both low iron cofactor availability and lack of OLE1 induction contribute to the drop in the UFA levels observed in mga2 $\Delta$ under iron-limited conditions. Collectively, these results strongly suggest that the relative UFA concentration in iron-deficient mga2 $\Delta$ cells lowers to levels that do not allow growth. 
A

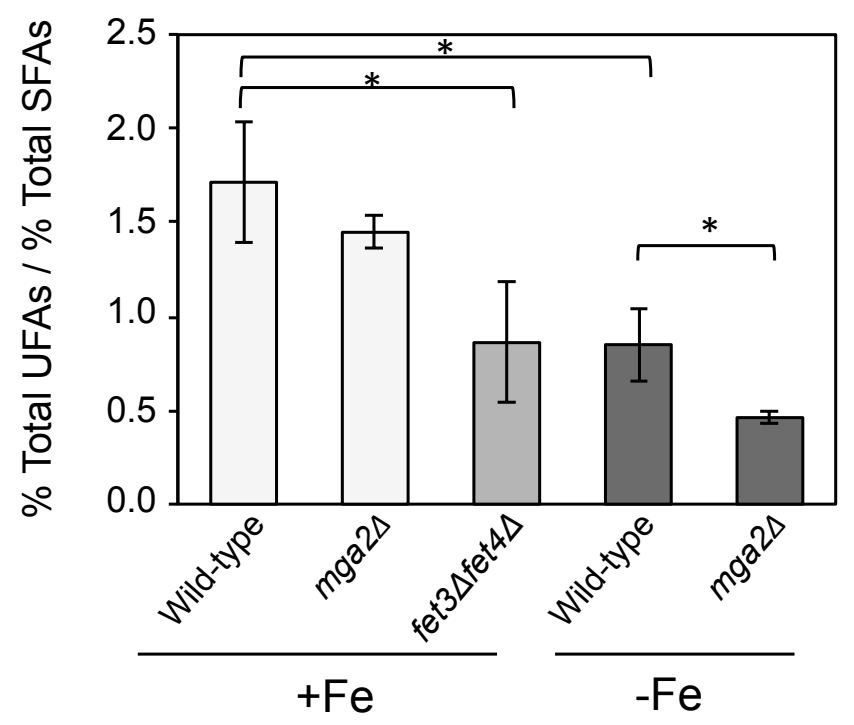

B

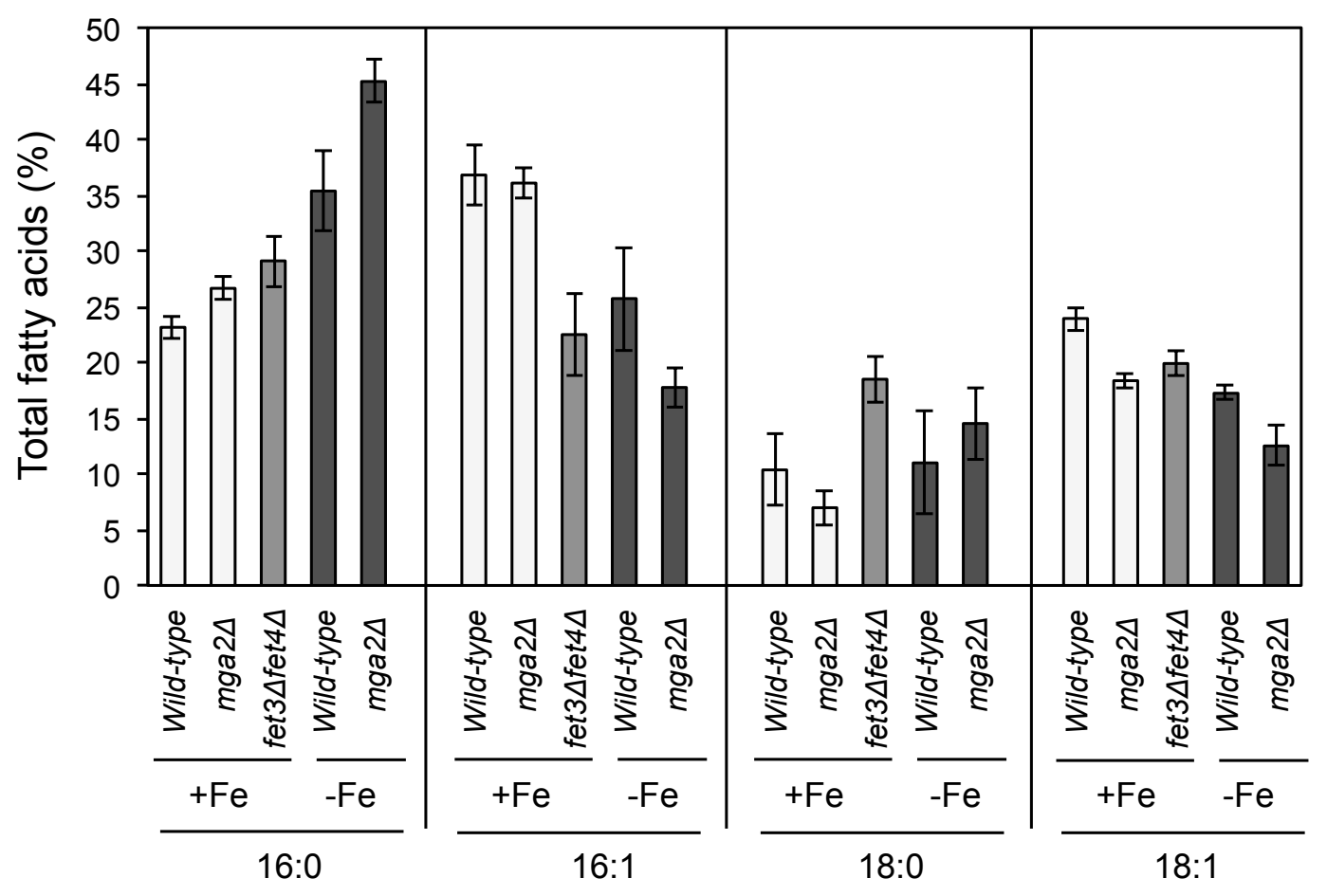

Figure 4. Effect of iron bioavailability and Mga2 on total fatty acid levels. The wild-type (BY4741) and $m g a 24$ (SPY824) cells were grown at $30^{\circ} \mathrm{C}$ for $8 \mathrm{~h}$ to the exponential phase in SC $(+\mathrm{Fe})$ or SC with $100 \mu \mathrm{M}$ BPS (-Fe). The fet34fet4 $(\mathrm{SPY} 386)$ 
mutant was also grown to the exponential phase in $\mathrm{SC}(+\mathrm{Fe})$. Total FAs were extracted and quantified as detailed in Material and Methods. (A) Iron and Mga2 improved UFA synthesis. The percentage of UFAs $(16: 1+18: 1)$ was represented in relation to SFAs $(14: 0+16: 0+18: 0)$. (B) The iron- and MGA2-deficient cells accumulated SFAs and showed diminished UFAs. Total palmitic (16:0), palmitoleic (16:1), stearic (18:0) and oleic (18:1) acids are separately represented. The results show the average and standard deviation of four biologically independent experiments. The asterisk (*) indicates statistically significant differences ( $p$-value $<0.05)$.

When we itemized the different SFA and UFA, we observed that under iron-deficient conditions, both the wild-type and mga2s cells specifically accumulated palmitic acid (16:0), which is an Ole1 substrate, and displayed lower palmitoleic acid levels (16:1), which is the product of palmitic acid desaturation (Figure 4B). A similar tendency was displayed by the fet3 $\Delta$ fet $4 \Delta$ strain under iron-sufficient conditions (Figure 4B). These results suggest that the bioavailability of iron limits yeast FA desaturase activity. More importantly, the iron-deficient mga2 $\Delta$ mutants accumulated more total palmitic acid (16:0) and less palmitoleic acid (16:1) than the wild-type strain (Figure 4B). These results suggest that $m g a 2 \Delta$ cells suffer a more severe FA desaturase defect than the wild-type cells when iron is not available. A similar tendency was observed when the levels of stearic (18:0) and oleic (18:1) acids were determined in the iron-sufficient and iron-deficient wild-type and mga2 $\Delta$ cells (Figure 4B). Although multiple interpretations are plausible, we considered that these results were consistent with the possibility that alterations in the molecular 
lipid packing, as a result of lowering UFA concentrations, would activate Mga2, and consequently OLE1 expression, in response to iron deficiency.

\subsection{OLE1 activation in response to iron deficiency requires the ubiquitin ligase Rsp5, Cdc48 ${ }^{\mathrm{Np} 14 / \mathrm{Ufd} 1}$ complex and the proteasome}

The Mga2 protein is tethered to the ER membrane through a carboxyterminal transmembrane (TM) helix domain. In response to low UFA or oxygen levels, Mga2 is cleaved from the ER membrane and enters the nucleus to activate OLE1 transcription (Hitchcock et al., 2001; Hoppe et al., 2000; Jiang et al., 2002). To inquire about the mechanism of OLE1 regulation by iron, we decided to investigate whether processing Mga2 was necessary to activate OLE1 upon iron limitation. We first determined the OLE1 transcript levels in the cells that expressed an Mga2 protein that lacked its ER-anchoring TM domain (MGA2ATM). As previously reported, deletion of the Mga2 TM segment caused the OLE1 mRNA levels to rise under normal growth conditions compared to a strain that expressed full length Mga2 (Figure 5A) (Chellappa et al., 2001). More importantly, iron depletion did not further augment OLE1 mRNA in the cells that expressed MGA2ATM (Figure 5A). Collectively, these results are consistent with a model in which iron deficiency would alter the composition of the ER membrane, which would facilitate Mga2 release and OLE1 transcription activation. 
A

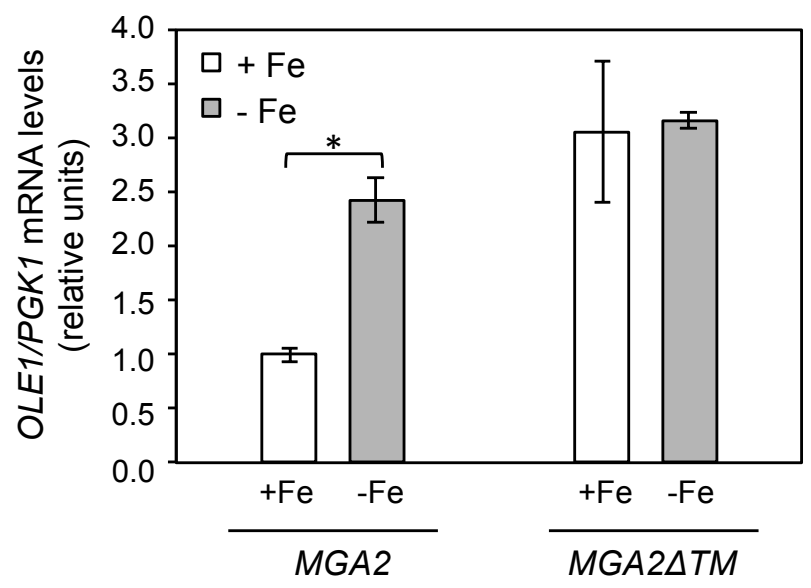

B

C
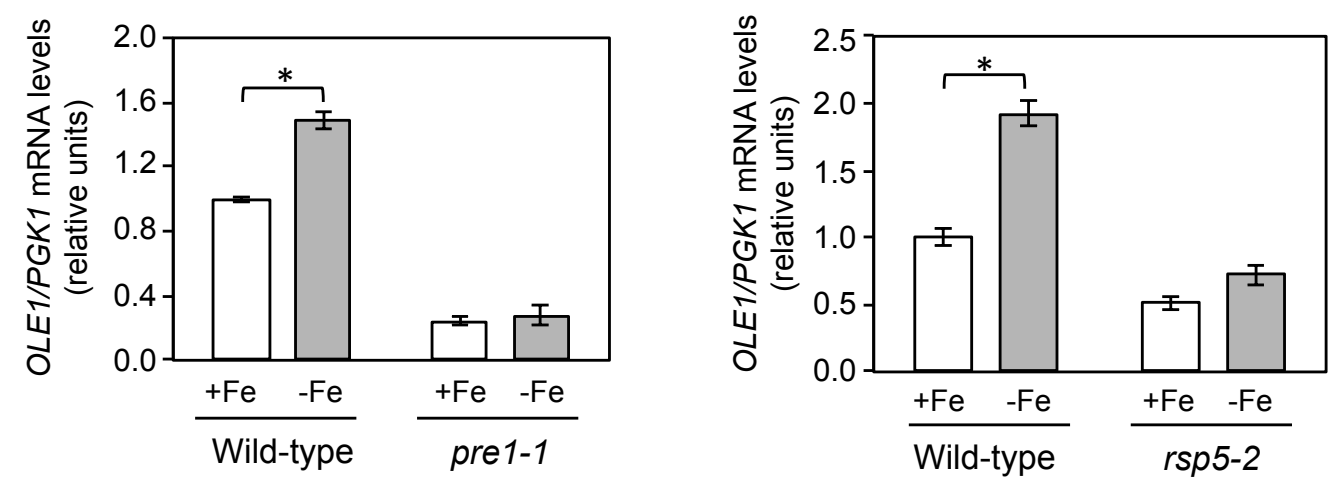

D

E
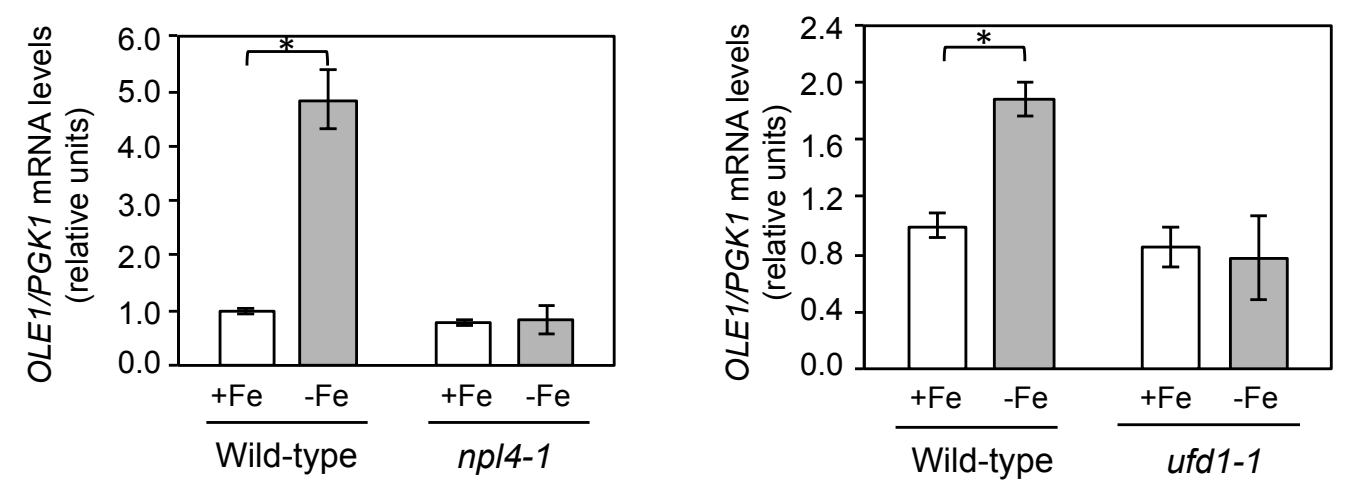

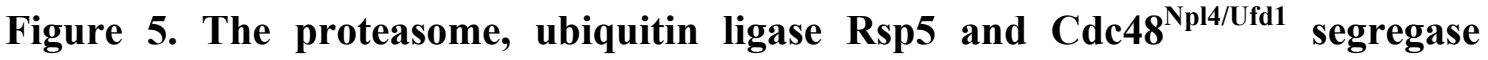
facilitate $O L E 1$ activation by low iron. (A) $O L E 1$ was not regulated by iron in the cells that expressed unanchored Mga2. The yeast mga24 (SPY824) cells transformed with MGA2 (pPS2364) or MGA2 $\triangle \mathrm{TM}$ (pPS2358) plasmids were grown and analyzed as described in Figure 1A. (B) The proteasome was required for OLE1 activation by low 
iron. The wild-type (YWO0607) and pre1-1 (YWO0608) cells were grown in SC at $25^{\circ} \mathrm{C}$ and then transferred to $37^{\circ} \mathrm{C}$. After $1 \mathrm{~h}$ at $37^{\circ} \mathrm{C}, 100 \mu \mathrm{M}$ BPS were added $(-\mathrm{Fe})$, or not $(+\mathrm{Fe})$, and cells were incubated for $5 \mathrm{~h}$ more. (C) E3 ubiquitin ligase Rsp5 was required to activate $O L E 1$ expression in response to low iron. The wild-type (Y0356) and rsp5-2 (Y0358) cells were grown as described in panel B. (D) Np14 facilitated OLE1 activation by low iron. The wild-type (PSY580) and npl4-1 (PSY2340) cells were grown in $\mathrm{SC}$ at $25^{\circ} \mathrm{C}$ and were then transferred to $30^{\circ} \mathrm{C}$. After $1 \mathrm{~h}$ at this temperature, $100 \mu \mathrm{M}$ BPS were added $(-\mathrm{Fe})$, or not $(+\mathrm{Fe})$, and cells were incubated for 5 h more. (E) Ufd1 enhanced OLE1 expression under low iron conditions. The wildtype (PSY580) and ufd1-1 (PSY3074) cells were grown as described in panel B. In all cases, total RNA was extracted and the OLE1 mRNA levels normalized with PGK1 mRNA were determined by RT-qPCR. Data indicate the average and standard deviation of three biologically independent experiments. The asterisk (*) indicates statistically significant differences ( $\mathrm{p}$-value $<0.05)$.

The release of Mga2 from the ER membrane in response to low UFA levels or low oxygen requires the proteasome, E3 ubiquitin ligase Rsp5 and segregase complex Cdc48 ${ }^{\text {Np14/Ufd1 }}$ (Hitchcock et al., 2001; Hoppe et al., 2000; Shcherbik et al., 2003). To determine the participation of these proteins in OLE1 activation by low iron, we used the temperature-permissive mutants of PRE1, RSP5, NPL4 and UFD1, which are essential genes in yeast. Whereas OLE1 transcript abundance increased in response to iron deficiency in all the wildtype strains, no OLE1 induction was observed for the pre1-1, rsp5-2, np/4-1 and ufd1-1 mutants at the non permissive temperature (Figure $5 \mathrm{~B}$ to $5 \mathrm{E}$ ). These 
results suggest that the proteasome, Rsp5 and $\mathrm{Cdc} 48^{\mathrm{Np} 14 / \mathrm{Ufd} 1}$ are required for Mga2-mediated transcriptional OLE1 activation by iron depletion.

\subsection{Transcriptional activation of MGA2 in response to iron deficiency favors OLE1 expression}

We decided to explore whether the expression of MGA2 was also regulated by iron bioavailability, as previously shown for OLE1 (Figure 1A). We observed that MGA2 mRNA levels increased upon addition of the $\mathrm{Fe}^{2+}$-chelator BPS or the deletion of FET3 and FET4 iron transporters (Figure 6A). Moreover, addition of excess iron to the iron-deficient medium decreased MGA2 transcript levels (Figure 6A, BPS+FAS). We used the Genomic Run-On approach to determine whether the up-regulation of the MGA2 mRNA by iron deficiency was mediated by changes in the transcription rate. We observed that both MGA2 TR and mRNA levels increased progressively while iron starvation advanced (Figure 6B). These results strongly suggest that MGA2 is transcriptionally activated in response to iron depletion.

To assess the relevance of MGA2 up-regulation in OLE1 desaturase activation, we compared OLE1 transcript abundance in yeast cells with different MGA2 expression levels. Specifically, we transformed mga2A cells with a centromeric (CEN) or a multicopy $(2 \mu)$ plasmid that contained MGA2. We corroborated that the multicopy plasmid rose MGA2 transcript levels would compare to the centromeric plasmid under both $+\mathrm{Fe}$ and $-\mathrm{Fe}$ conditions (Figure 6C). In both cases, MGA2 was up-regulated when iron was depleted from the growth medium (Figure $6 \mathrm{C}$ ). It was noteworthy that the cells which 
overexpressed MGA2 exhibited higher OLE1 expression levels than the cells that expressed centromeric MGA2 (Figure 6D). These results indicate that increasing MGA2 expression favors OLE1 up-regulation.

A

B
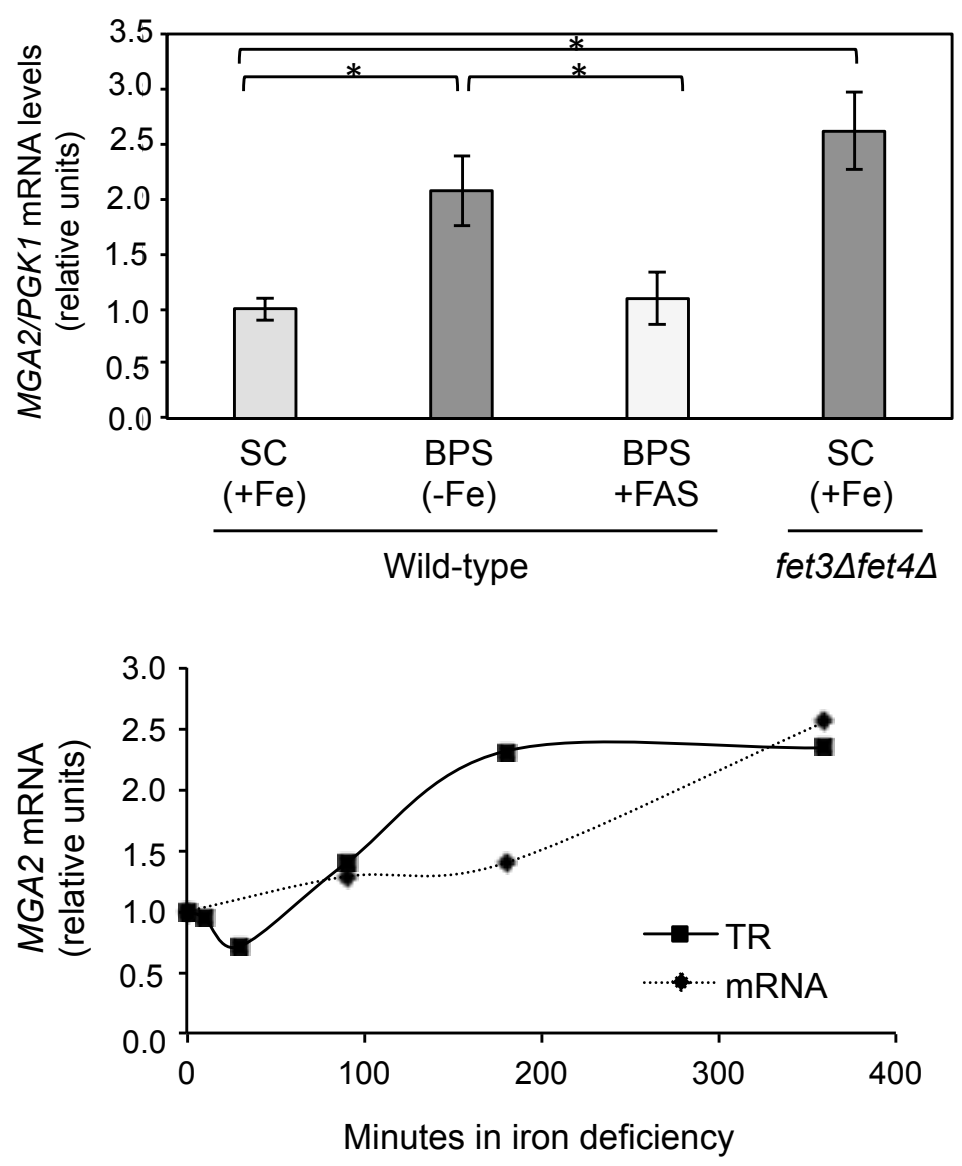

C

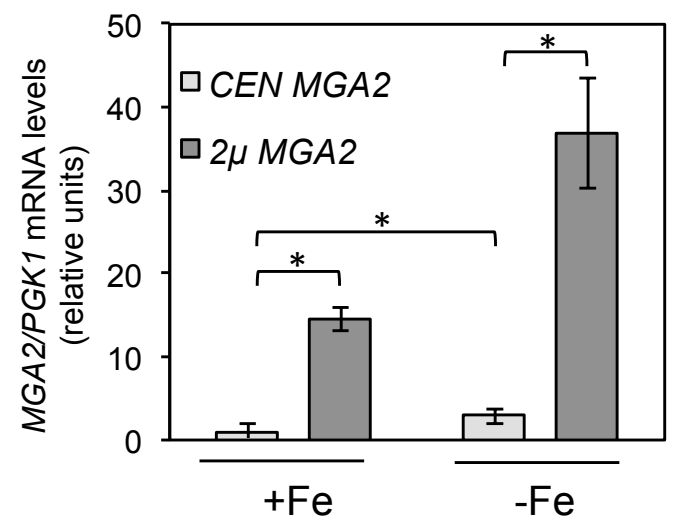

D

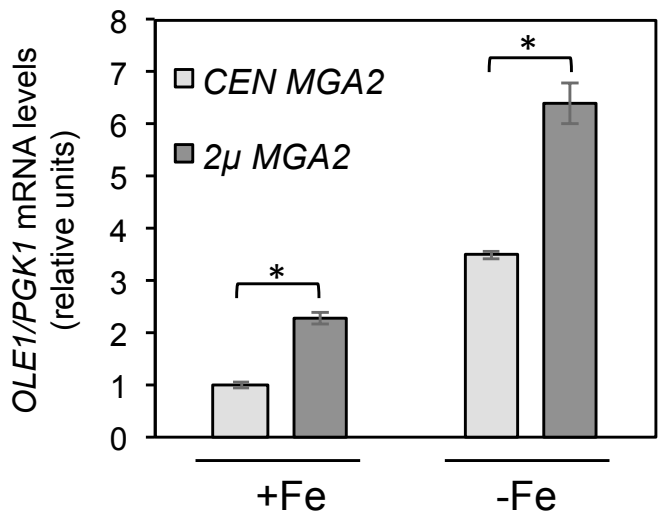


Figure 6. Regulation of the $M G A 2$ mRNA levels by iron bioavailability. (A) $M G A 2$ mRNA levels increased in response to nutritional and genetic iron deficiency. The wildtype (BY4741) and fet34fet4ム (SPY386) cells were grown as described in Figure 1A. Total RNA was extracted, and the $M G A 2$ mRNA levels were determined by RT-qPCR. The $M G A 2$ transcript levels were normalized with $P G K 1$ mRNA. (B) Iron deficiency increased the MGA2 transcription rate. The wild-type (HTLU-2832-1B) cells were grown as described in Figure 1B. Then samples were processed to determine the $M G A 2$ transcript levels (mRNA) by RT-qPCR and the MGA2 transcription rate (TR) by GRO. (C and D) $M G A 2$ overexpression caused $O L E 1$ up-regulation. The yeast mga2A (SPY824) cells transformed with either a centromeric (pPS2369, CEN) or a multicopy (YEplac181-3HA-MGA2, 2 $\mu$ ) plasmid that expressed $M G A 2$ were grown for $6 \mathrm{~h}$ in SC $(+\mathrm{Fe})$ or $\mathrm{SC}+100 \mu \mathrm{M}$ BPS $(-\mathrm{Fe})$. Total RNA was extracted, and the $M G A 2$ (panel B) and OLE1 (panel C) mRNA levels were determined by RT-qPCR. The results indicate the average and standard deviation of three biologically independent experiments. The asterisk $(*)$ indicates statistically significant differences ( $\mathrm{p}$-value $<0.05$ ).

\subsection{Mga2 activates its own expression in response to iron deficiency, cold, hypoxia and a drop in unsaturated fatty acids}

To investigate the mechanism of MGA2 regulation, we ascertained whether iron-regulated transcription factors Aft1 and Aft2 were implicated in MGA2 activation by iron depletion. We observed that MGA2 up-regulation still occurred in an aft1 $\Delta$ aft2 $\Delta$ mutant, which suggests Aft1- and Aft2-independent mechanisms (Figure 7A). As previously observed for OLE1, addition of linoleic acid abrogated MGA2 regulation by iron (Figure 7B). As MGA2 seems to be 
regulated by iron and unsaturated fatty acid availability, we wondered whether this was also the case for low-oxygen and low-temperature conditions. As shown in Figure $7 \mathrm{C}$, both the OLE1 and MGA2 mRNAs were up-regulated in response to hypoxia and cold. Since the Mga2 protein activated OLE1 in response to iron depletion, we ascertained whether Mga2 also activated its own transcription under these conditions. To address this question, we fused the MGA2 promoter region to the lacZ reporter gene $\left(P_{M G A 2}-l a c Z\right)$ and determined $\beta$-galactosidase activity in the wild-type and mga2 $\Delta$ cells cultivated under $+\mathrm{Fe}$ and $-\mathrm{Fe}$ conditions. As expected from the MGA2 TR data (Figure 6A), $P_{M G A 2^{-}}$ lacZ led to increased $\beta$-galactosidase activity when the wild-type cells were deprived of iron (Figure 7D). More importantly, the $P_{M G A 2}-$ lacZ activation by low iron did not occur in the mga2 $\Delta$ cells (Figure 7D), which strongly suggests that Mga2 activates its own transcription upon iron limitation. To address whether MGA2 auto-activation is a specific response to iron depletion or, instead, a more general regulatory mechanism to enhance OLE1 activation under other conditions, we studied $P_{M G A 2-l a c Z}$ expression in both the wild-type and mga2 $\Delta$ cells grown at low temperature $\left(10^{\circ} \mathrm{C}\right)$, low oxygen or in the presence of linoleic acid (18:2). As previously shown for OLE1 (Chellappa et al., 2001; Hoppe et al., 2000; Kwast et al., 1999; Nakagawa et al., 2002; Vasconcelles et al., 2001), $P_{M G A 2-l a c Z}$ expression increased in response to cold and hypoxic treatments, and decreased when UFAs were added (Figures 7D and 7E). Moreover, no remarkable $P_{M G A 2}-l a c Z$ regulation was observed in the mga2 $\Delta$ mutant cells. Altogether these results strongly indicate that Mga2 activates the expression of both genes MGA2 and OLE1 in response to multiple conditions that alter the FA composition, including low UFAs, temperature, oxygen and iron (Figure 8). 
A

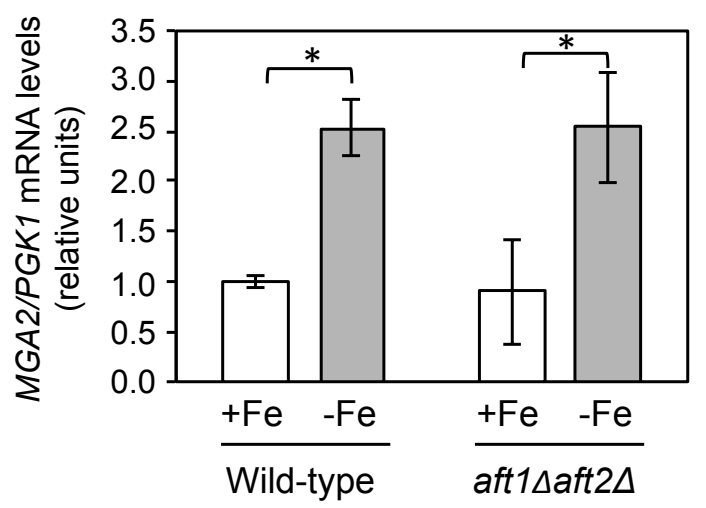

C

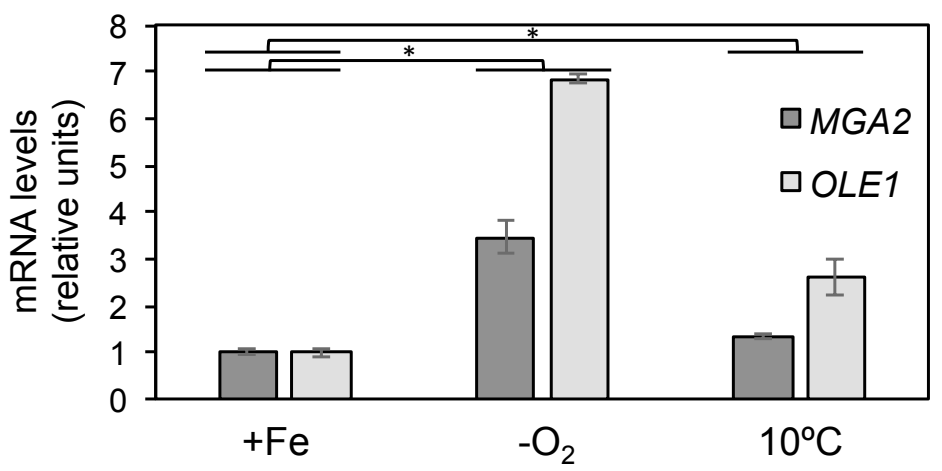

$\mathrm{D}$

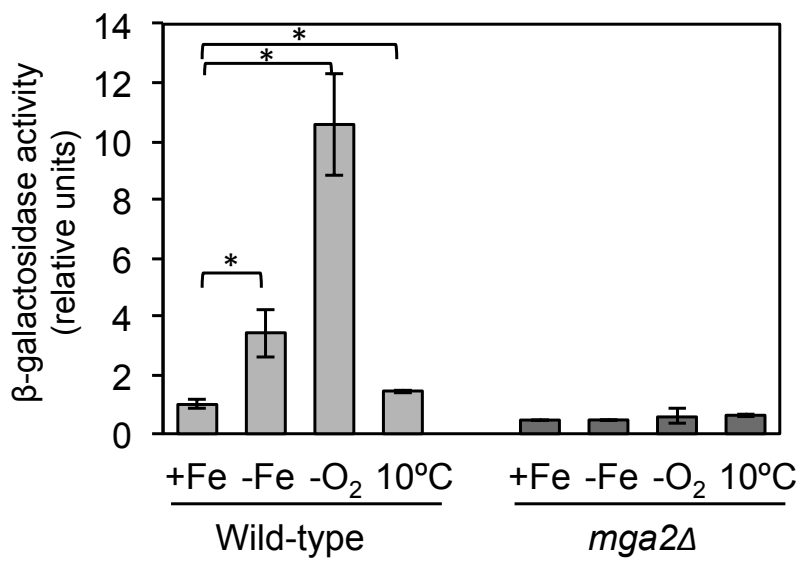

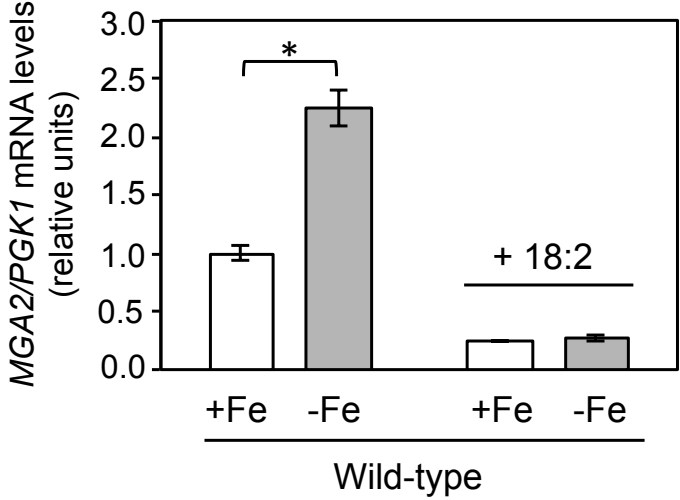

$E$

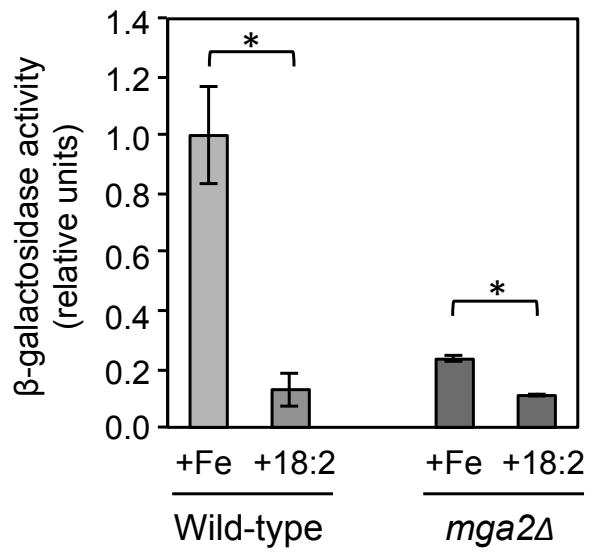

Figure 7. Mga2 activates its own expression in response to low iron, cold, hypoxia and low UFAs. (A) Aft1 and Aft2 were not responsible for MGA2 activation by iron depletion. The wild-type (W303-1A) and aft14aft24 (MML1088) cells were grown as 
in Figure 1C. (B) Iron deficiency did not activate $M G A 2$ in the presence of linoleic acid. The wild-type (BY4741) cells were grown for $6 \mathrm{~h}$ in $\mathrm{SC}(+\mathrm{Fe})$ or $\mathrm{SC}+100 \mu \mathrm{M}$ BPS (Fe), without or with $1 \mathrm{mM}$ linoleic acid (+18:2). (C) A drop in the oxygen levels or temperature activated $M G A 2$ expression. The wild-type (BY4741) cells were grown at $30^{\circ} \mathrm{C}$ to the exponential phase in $\mathrm{SC}(+\mathrm{Fe})$ and were then transferred to hypoxia for $5 \mathrm{~h}$ $\left(-\mathrm{O}_{2}\right)$ or $10^{\circ} \mathrm{C}$ for $1 \mathrm{~h}$. (D) Hypoxia and cold activated $M G A 2$ expression in an Mga2dependent manner. The wild-type (BY4741) and mga24 (SPY824) cells transformed with the pSP1039 ( $P_{M G A 2}$-lacZ) plasmid were grown as in panel C. (E) A drop in the UFA levels activated $M G A 2$ expression in an Mga2-dependent manner. The wild-type (BY4741) and mga2A (SPY824) cells transformed with the pSP1039 (P $P_{M G A 2}$-lacZ) plasmid were grown in the SC $(+\mathrm{Fe})$ or SC that contained $1 \mathrm{mM}$ linoleic acid for $6 \mathrm{~h}$ $(+18: 2)$. Either total RNA was extracted and the $M G A 2$ mRNA levels determined by RT-qPCR (panels A, B and C), or $\beta$-galactosidase assays were performed (panels D and E). Data represent the average and standard deviation of three biologically independent experiments. The asterisk $(*)$ indicates statistically significant differences (p-value $<$ $0.05)$.

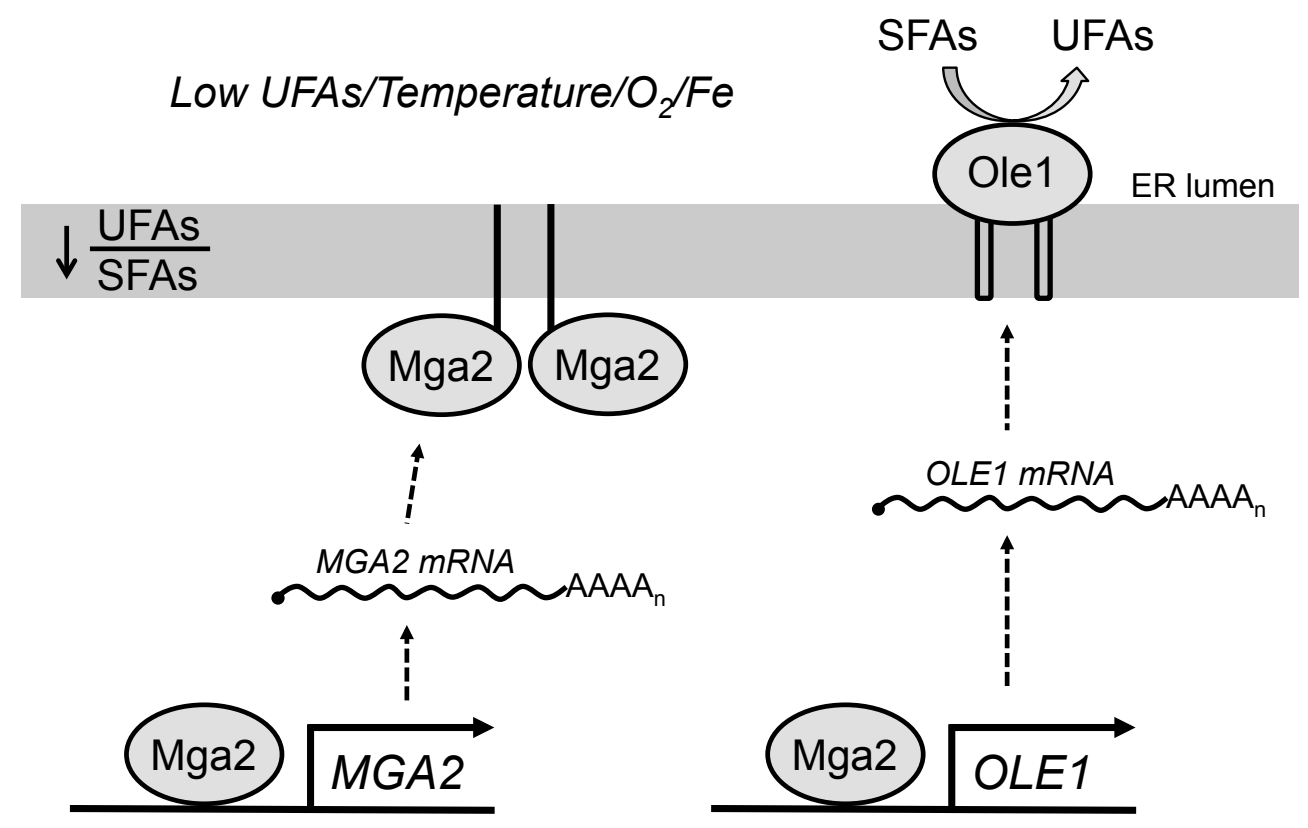


Figure 8. A model for $O L E 1$ transcriptional activation in response to iron deficiency. Upon iron deficiency, yeast cells diminished the availability of this cofactor for multiple iron-dependent processes, including the Ole1 $\Delta 9$-FA desaturase enzyme. As a direct consequence, the proportion of UFAs/SFAs lowered, which probably changed ER membrane fluidity and enhanced Mga2 processing. Released Mga2 translocated to the nucleus and activated $M G A 2$ expression, which contributed to increase the pool of the available Mga2 transcription factor. Finally, Mga2 activated the transcription of $O L E 1$, which helped to increase the UFA levels. 


\section{Discussion}

Oxygen and iron are indispensable for the catalytic activity of eukaryotic $\Delta 9$-FA desaturases. By studying yeast Ole1, multiple groups have characterized the mechanisms that activate the expression of this $\Delta 9-\mathrm{FA}$ desaturase in response to low oxygen levels (Jiang et al., 2001; Jiang et al., 2002; Nakagawa et al., 2002). More recent data support the notion that iron defects also activate OLE1 expression. For instance, addition of $\mathrm{Fe}^{2+}$ chelators to the growth medium increases OLE1 transcript abundance (Chellappa et al., 2001; Puig et al., 2005). Moreover, yeast cells that lack monothiol glutaredoxin GRX5, which functions in mitochondrial iron-sulfur cluster biosynthesis, upregulate the expression of a group of genes, including the iron regulon and OLE1 (Belli et al., 2004). Finally, it is also well-known that addition of cobalt, which induces the iron regulon, enhances OLE1 expression (Kwast et al., 1999; Stadler and Schweyen, 2002; Vasconcelles et al., 2001). We showed here that the addition of excess iron to a medium that contained BPS decreased OLE1 expression to basal levels (Figure 1A). A previous study indicated that the deletion of the AFT1 transcription factor activates the expression of various genes related to oxygen and lipid metabolism probably in an Mga2-dependent

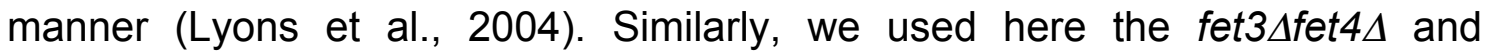
aft $1 \Delta$ aft2 $\Delta$ yeast mutants to show that genetic iron deficiencies activate OLE1 expression (Figure 1A and 1E). By using GRO, we demonstrated that OLE1 was activated at the transcriptional level in response to iron depletion (Figure 1D). As previously reported for other conditions including hypoxia and low temperature (Chellappa et al., 2001; Jiang et al., 2001; Jiang et al., 2002; Nakagawa et al., 2002), Mga2 was also the main factor to activate OLE1 upon 
iron limitation (Figure 2). However, our data cannot ignore the contribution of Spt23 since a subtle drop in OLE1 up-regulation by low iron was still observed in the spt23A mutants. As proposed for other stresses (Ballweg and Ernst, 2017), crosstalk between both transcription factors could occur in iron deficiency with Mga2 functioning as the main OLE1 regulator and Spt23 being responsible for fine-tuning the response.

We determined the levels of total SFAs and UFAs in the wild-type cells grown under normal conditions and in iron-deficient cells, which were achieved

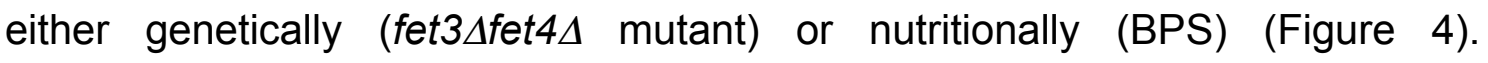
Consistently with Ole1 being an iron-dependent enzyme, when the iron bioavailability of the wild-type cells was restricted the proportion of UFAs lowered. Specifically, we observed that iron deprivation led total palmitic acid to accumulate (16:0), and UFAs palmitoleic (16:1) and oleic (18:1) acid abundance to decrease. However, a previous study has not reported significant alterations in the SFAs and UFAs levels under low iron conditions (ShakouryElizeh et al., 2010). This discrepancy could be due to the different treatment used to limit iron availability. Our iron-deficient conditions consisted in cultivating yeast cells for $6 \mathrm{~h}$ in a medium supplemented with $100 \mu \mathrm{M}$ of the $\mathrm{Fe}^{2+}$-specific chelator BPS, which dramatically limits extracellular available iron. However, those authors did not add an iron chelator but, instead, they cultivated cells for $24 \mathrm{~h}$ in a defined-iron medium that contained $5 \mu \mathrm{M}$ of ferrous iron (-Fe condition) compared to $300 \mu \mathrm{M}$ of ferrous iron (+Fe conditions). In agreement with a mild iron-deficient condition, they did not observe any changes in Ole1 expression (Shakoury-Elizeh et al., 2010), whereas the more severe iron depletion that we used activated Mga2 and OLE1 expression (Figure 1). 
Despite the drop in total UFAs displayed by the wild-type cells under iron-limited conditions, yeast still synthesized sufficient UFAs for grow (Figures 3 and 4). However, deletion of MGA2 further diminished the synthesis of UFAs under the low iron conditions to levels that blocked growth (Figure 3 and 4). These data demonstrated that the mga2 $\Delta$ growth defect in low iron was due to the lack of UFAs as it can be recued by adding external UFAs or OLE1 expression with a galactose-regulated promoter (Figure 3). Furthermore, these results suggested that the OLE1 up-regulation by low iron partially compensated the drop in iron availability and, therefore, Ole1 activity.

The Mga2 and Ole1 role during the progress of iron deficiency could be divided into various stages. In an initial stage, Mga2 would not be activated and no increase in OLE1 expression would be observed. In response to more severe iron depletion, Mga2 would activate OLE1 expression. A two-stages situation has been described for Mga2 during the response to oxidative stress (Kelley and Ideker, 2009). By using a genome-wide approach, these authors uncovered that Mga2 transcription factor was important for the adaptive, but not the acute, response to hydrogen peroxide by activating the expression of genes implicated in the ergosterol, the FA and the zinc metabolic pathways (Kelley and Ideker, 2009). Additional evidence that connect zinc and lipid homeostasis has shown that the Mga2 transcription factor activates the expression of $I Z H 2$ and IZH4 in response to excess zinc (Lyons et al., 2004). Both genes display homology to vertebrate membrane steroid receptors, and are regulated by oxygen and fatty acid levels (Lyons et al., 2004).

Regarding the mechanism that activates Mga2 and, consequently OLE1 expression, in response to iron deprivation, multiple data shown herein 
suggested that changes in the proportion of FAs could cause alterations in the lipid packing of the ER membrane that promote Mga2 processing and OLE1 transcription, as previously shown for hypoxia and low temperatures (Jiang et al., 2002; Jiang et al., 2001; Nakagawa et al., 2002). First, addition of extracellular UFAs eliminated OLE1 activation by iron depletion (Figure 2A). Second, deletion of the TM helix, which tethered Mga2 to the ER membrane, prevented OLE1 iron regulation (Figure 5A). Third, the proteasome, E3 ubiquitin ligase Rsp5 and the Npl4 and Ufd1 components of the Cdc48 ${ }^{\mathrm{Np} 14 / \mathrm{Ufd} 1}$ segregase complex were necessary for OLE1 up-regulation by iron deprivation (Figure 5).

Here we have shown that yeast cells activate the transcription of OLE1 in response to iron deprivation (Figure 1D). In the absence of $M G A 2$, yeast cells are unable to enhance the recruitment of the RNA pol II to the OLE1 promoter region and do not increase OLE1 mRNA levels when iron is scarce (Figure 2). These results highlight the relevance of Mga2 transcriptional activity on the upregulation of OLE1 by iron limitation. However, post-transcriptional regulatory mechanisms could also contribute to the final Ole1 protein levels and activity. The steady-state levels of each mRNA depend directly on its TR and inversely on its decay rates. By using the GRO approach, we have simultaneously determined the experimental values for the TR and the mRNA levels of OLE1 (Figure 1D). From these empirical data, we can estimate the decay rate of the OLE1 mRNA at each time-point (Garcia-Martinez et al., 2004). Although both OLE1 TR and mRNA levels increase during the progress of the iron deficiency, its pattern is not completely parallel, which suggests that a post-transcriptional contribution to the final OLE1 transcript levels exists. Previous results have shown that Mga2 also regulates OLE1 mRNA stability according to the 
availability of fatty acids (Kadasamy et al., 2004). Mga2 seems to play a stabilization function on OLE1 transcript in FA-free media and a destabilization effect when the medium is supplemented with UFAs (Kadasamy et al., 2004). Moreover, in response to iron depletion, yeast cells express a tandem zincfinger protein called Cth2 that binds to mRNAs, which contain AU-rich elements within their 3'-untranslated region, and promotes their turnover (Puig et al., 2005; Pedro-Segura et al., 2008). We have previously shown that the OLE1 transcript contains two putative AU-rich elements and its mRNA levels increase in cells lacking CTH2 (Puig et al., 2005). These observations suggest that various regulatory factors, including Mga2 and Cth2, could control OLE1 mRNA stability during the progress of iron deficiency.

Our data also uncovered that MGA2 expression increased in response to multiple stresses, including changes in UFAs in the growth medium, a drop in temperature and alterations in oxygen or iron availability. Furthermore, MGA2 up-regulation by low iron did not occur when excess UFAs were added to the growth medium (Figure 7B). These observations suggest that changes in the composition and fluidity of lipid membranes could also be responsible for MGA2 activation. As the Mga2 transcription factor is processed and activated in response to these stresses (Hoppe et al., 2000; Piwko and Jentsch, 2006), we postulated that the Mga2 protein could activate the expression of its own mRNA in an auto-activation mechanism. Our $\beta$-galactosidase assays run with the mga2 $\Delta$ cells that contained a $P_{M G A 2-l a c Z}$ construct confirmed this hypothesis, and our overexpression experiments corroborated that an increasing MGA2 expression contributed to OLE1 activation. 
The data presented herein indicated that a decrease in the bioavailability of iron caused a drop in Ole1 activity, which led to changes in the lipid composition of membranes. Consequently, Mga2 regulatory factor was released from the ER membrane, traveled to the nucleus, and activated OLE1 transcription to compensate the unsaturated/saturated fatty acid imbalance. Furthermore, our study revealed that Mga2 activated its own expression in response to multiple inputs (Figure 8). This novel regulatory mechanism could contribute to help yeast cells adapt to hypoxia, cold, low UFA concentrations and iron deficiency. 


\section{Acknowledgments}

This work was supported by a predoctoral contract from the Spanish Ministry of Economy, Industry and Competitiveness to Antonia M. Romero, and the Spanish Ministry of Economy, Industry and Competitiveness grants BIO2014-56298-P and BIO2017-87828-C2-1-P and FEDER funds to Sergi Puig. We are grateful to Pilar Miró, Dr. José Enrique Perez-Ortín, Dr. Paula Alepuz, and the members of the Iron Homeostasis laboratory for scientific comments and technical assistance. We are also in debt with Drs. Fred Cross, Enrique Herrero, Dieter H. Wolf, Stefan Jentsch, Florian Paasch, Pamela Silver and Richard S. Zitomer for providing yeast strains and plasmids used in this study.

Conflict of interest: The authors declare that no conflict of interest exists. 


\section{References}

Adiphatla, R.M., and Hatcher, J.F. (2007). Role of lipids in brain injury and diseases. Future Lipidol 2, 403-422.

Aguilar, P.S., and de Mendoza, D. (2006). Control of fatty acid desaturation: a mechanism conserved from bacteria to humans. Mol Microbiol 62, 1507-1514.

Ballweg, S., and Ernst, R. (2017). Control of membrane fluidity: the OLE pathway in focus. Biol Chem 398, 215-228.

Belli, G., Molina, M.M., Garcia-Martinez, J., Perez-Ortin, J.E., and Herrero, E. (2004). Saccharomyces cerevisiae glutaredoxin 5-deficient cells subjected to continuous oxidizing conditions are affected in the expression of specific sets of genes. J Biol Chem 279, 12386-12395.

Borrull, A., Poblet, M., and Rozès, N. (2015). New insights into the capacity of commercial wine yeasts to grow on sparkling wine media. Factor screening for improving wine yeast selection. Food Microbiol 48, 41-48.

Chellappa, R., Kandasamy, P., Oh, C.S., Jiang, Y., Vemula, M., and Martin, C.E. (2001). The membrane proteins, Spt23p and Mga2p, play distinct roles in the activation of Saccharomyces cerevisiae $O L E 1$ gene expression. Fatty acid-mediated regulation of Mga2p activity is independent of its proteolytic processing into a soluble transcription activator. J Biol Chem 276, 43548-43556.

Covino, R., Ballweg, S., Stordeur, C., Michaelis, J.B., Puth, K., Wernig, F., Bahrami, A., Ernst, A.M., Hummer, G., and Ernst, R. (2016). A Eukaryotic Sensor for Membrane Lipid Saturation. Mol Cell 63, 49-59.

Garcia-Martinez, J., Aranda, A., and Perez-Ortin, J.E. (2004). Genomic run-on evaluates transcription rates for all yeast genes and identifies gene regulatory mechanisms. Mol Cell 15, 303-313.

Gomar-Alba, M., Alepuz, P., and del Olmo, M. (2013). Dissection of the elements of osmotic stress response transcription factor Hot1 involved in the interaction with MAPK Hog1 and in the activation of transcription. Biochim Biophys Acta 1829, 1111-1125.

Hitchcock, A.L., Krebber, H., Frietze, S., Lin, A., Latterich, M., and Silver, P.A. (2001). The conserved npl4 protein complex mediates proteasome-dependent membranebound transcription factor activation. Mol Biol Cell 12, 3226-3241.

Hoppe, T., Matuschewski, K., Rape, M., Schlenker, S., Ulrich, H.D., and Jentsch, S. (2000). Activation of a membrane-bound transcription factor by regulated ubiquitin/proteasome-dependent processing. Cell 102, 577-586.

Jiang, Y., Vasconcelles, M.J., Wretzel, S., Light, A., Gilooly, L., McDaid, K., Oh, C.S., Martin, C.E., and Goldberg, M.A. (2002). Mga2p processing by hypoxia and 
unsaturated fatty acids in Saccharomyces cerevisiae: impact on LORE-dependent gene expression. Eukaryot Cell 1, 481-490.

Jiang, Y., Vasconcelles, M.J., Wretzel, S., Light, A., Martin, C.E., and Goldberg, M.A. (2001). MGA2 is involved in the low-oxygen response element-dependent hypoxic induction of genes in Saccharomyces cerevisiae. Mol Cell Biol 21, 6161-6169.

Kadasamy, P., Vemula, M., Oh, C.S., Chellappa, R., and Martin, C.E. (2004). Regulation of unsaturated fatty acid biosynthesis in Saccharomyces: the endoplasmic reticulum membrane protein, Mga2p, a transcriptional activator of the OLE1 gene, regulates the stability of the OLE1 mRNA through exosomemediated mechanisms. J Biol Chem 279, 36586-36592.

Kaplan, C.D., and Kaplan, J. (2009). Iron acquisition and transcriptional regulation. Chem Rev 109, 4536-4552.

Kelley, R., and Ideker, T. (2009). Genome-wide fitness and expression profiling implicate Mga2 in adaptation to hydrogen peroxide. PLoS Genetics 5, e1000488.

Kolawa, N., Sweredoski, M.J., Graham, R.L., Oania, R., Hess, S., and Deshaies, R.J. (2013). Perturbations to the ubiquitin conjugate proteome in yeast deltaubx mutants identify Ubx2 as a regulator of membrane lipid composition. Mol Cell Proteomics 12, 2791-2803.

Kushnirov, V.V. (1998). Rapid and reliable protein extraction from yeast. Yeast 16, 857-860.

Kwast, K.E., Burke, P.V., Staahl, B.T., and Poyton, R.O. (1999). Oxygen sensing in yeast: evidence for the involvement of the respiratory chain in regulating the transcription of a subset of hypoxic genes. Proc Natl Acad Sci USA 96, 5446-5451.

Kwast, K.E., Lai, L.C., Menda, N., James III, D.T., Aref, S., and Burke, P.V. (2002). Genomic analyses of anaerobically induced genes in Saccharomyces cerevisiae: functional roles of Rox1 and other factors in mediating the anoxic response. J. Bacteriol 18, 250-265.

Longtine, M.S., Mckenzie, A., Demarini, D.J., Shah, N.G., Wach, A., Brachat, A., Philippsen, P., and Pringle, J.R. (1998). Additional modules for versatile and economical PCR-based gene deletion and modification in Saccharomyces cerevisiae. Yeast 14, 953-961.

Lyons, T.J., Villa, N.Y., Regalla L.M., Kupchak, B.R., Vagstad, A., and Eide, D.J. (2004). Metalloregulation of yeast membrane steroid receptor homologs. Proc Natl Acad Sci USA 101, 5506-5511.

Martin, C.E., Oh, C.S., and Jiang, Y. (2007). Regulation of long chain unsaturated fatty acid synthesis in yeast. Biochim Biophys Acta 1771, 271-285. 
Nakagawa, Y., Sakumoto, N., Kaneko, Y., and Harashima, S. (2002). Mga2p is a putative sensor for low temperature and oxygen to induce OLE1 transcription in Saccharomyces cerevisiae. Biochem Biophys Res Commun 291, 707-713.

Pedro-Segura, E., Vergara, S.V., Rodríguez-Navarro. S., Parker, R., Thiele, D.J., and Puig S. (2008). The Cth2 ARE-binding protein recruits the Dhh1 helicase to promote the decay of succinate dehydrogenase SDH4 mRNA in response to iron deficiency. J Biol Chem 283, 28527-28535.

Piwko, W., and Jentsch, S. (2006). Proteasome-mediated protein processing by bidirectional degradation initiated from an internal site. Nat Struct Mol Biol 13, 691-697.

Puig, S., Askeland, E., and Thiele, D.J. (2005). Coordinated remodeling of cellular metabolism during iron deficiency through targeted mRNA degradation. Cell 120, 99-110.

Puig, S., Lau, M., and Thiele, D.J. (2004). Cti6 is an Rpd3-Sin3 histone deacetylaseassociated protein required for growth under iron-limiting conditions in Saccharomyces cerevisiae. J Biol Chem. 279, 30298-30306.

Rape, M., Hoppe, T., Gorr, I., Kalocay, M., Richly, H., and Jentsch, S. (2001). Mobilization of processed, membrane-tethered SPT23 transcription factor by CDC48(UFD1/NPL4), a ubiquitin-selective chaperone. Cell 107, 667-677.

Samanfar, B., Omidi, K., Hooshyar, M., Laliberte, B., Alamgir, M., Seal, A.J., AhmedMuhsin, E., Viteri, D.F., Said, K., Chalabian, F., et al. (2013). Large-scale investigation of oxygen response mutants in Saccharomyces cerevisiae. Mol Biosyst 9, 1351-1359.

Sanvisens, N., Bañó, M.C., Huang, M., and Puig, S. (2011). Regulation of ribonucleotide reductase in response to iron deficiency. Mol Cell 44, 759-769.

Sanvisens, N., and Puig, S. (2011). Causes and consequences of nutritional iron deficiency in living organisms. In Biology of starvation in humans and other organisms, T.C. Merkin, ed. (Nova Science Publishers), pp. 245-276.

Sanvisens, N., Romero, A.M., An, X., Zhang, C., de Llanos, R., Martínez-Pastor, M.T., Bañó, M.C., Huang, M., and Puig, S. (2014). Yeast Dun1 kinase regulates ribonucleotide reductase inhibitor Sml1 in response to iron deficiency. Mol Cell Biol 34, 3259-3271.

Shakoury-Elizeh, M., Protchenko, O., Berger, A., Cox, J., Gable, K., Dunn, T.M., Prinz, W.A., Bard, M., and Philpott, C.C. (2010). Metabolic response to iron deficiency in Saccharomyces cerevisiae. J Biol Chem 285, 14823-14833.

Shcherbik, N., and Haines, D.S. (2007). Cdc48p(Npl4p/Ufd1p) binds and segregates membrane-anchored/tethered complexes via a polyubiquitin signal present on the anchors. Mol Cell 25, 385-397. 
Shcherbik, N., Zoladek, T., Nickels, J.T., and Haines, D.S. (2003). Rsp5p is required for ER bound Mga2p120 polyubiquitination and release of the processed/tethered transactivator Mga2p90. Curr Biol 13, 1227-1233.

Singh, P. (2017). Budding Yeast: An Ideal Backdrop for In vivo Lipid Biochemistry. Front Cell Dev Biol 4, 156.

Stadler, J.A., and Schweyen, R.J. (2002). The yeast iron regulon is induced upon cobalt stress and crucial for cobalt tolerance. J Biol Chem 277, 39649-39654.

Surma, M.A., Klose, C., Peng, D., Shales, M., Mrejen, C., Stefanko, A., Braberg, H., Gordon, D.E., Vorkel, D., Ejsing, C.S., et al. (2013). A lipid E-MAP identifies Ubx2 as a critical regulator of lipid saturation and lipid bilayer stress. Mol Cell 51, 519-530.

Ter Linde, J.J.M. and Steensma, H.Y. (2002). A microarray-assisted screen for potential Hap1 and Rox1 target genes in Saccharomyces cerevisiae. Yeast 19, 825840.

Vasconcelles, M.J., Jiang, Y., McDaid, K., Gilooly, L., Wretzel, S., Porter, D.L., Martin, C.E., and Goldberg, M.A. (2001). Identification and characterization of a low oxygen response element involved in the hypoxic induction of a family of Saccharomyces cerevisiae genes. Implications for the conservation of oxygen sensing in eukaryotes. J Biol Chem 276, 14374-14384.

Zhang, S., Skalsky, Y., and Garfinkel, D.J. (1999). MGA2 or SPT23 is required for transcription of the delta9 fatty acid desaturase gene, OLE1, and nuclear membrane integrity in Saccharomyces cerevisiae. Genetics 151, 473-483. 